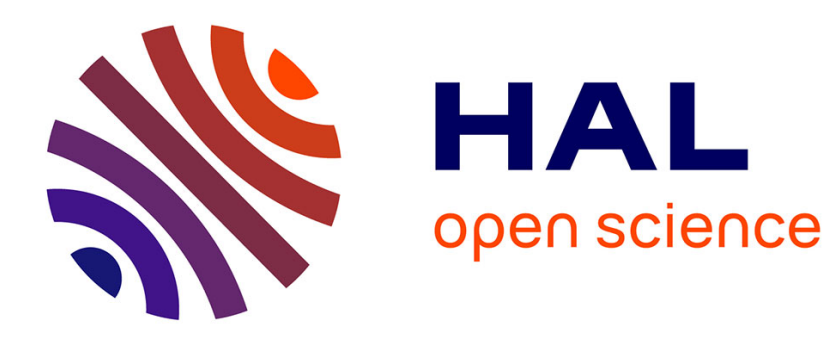

\title{
The asymptotic behaviour of solutions of the forced Burgers equation on the circle
}

Patrick Bernard

\section{To cite this version:}

Patrick Bernard. The asymptotic behaviour of solutions of the forced Burgers equation on the circle. Nonlinearity, 2005, 18, pp.101-124. 10.1088/0951-7715/18/1/006 . hal-01251197

\section{HAL Id: hal-01251197 \\ https://hal.science/hal-01251197}

Submitted on 6 Jan 2016

HAL is a multi-disciplinary open access archive for the deposit and dissemination of scientific research documents, whether they are published or not. The documents may come from teaching and research institutions in France or abroad, or from public or private research centers.
L'archive ouverte pluridisciplinaire HAL, est destinée au dépôt et à la diffusion de documents scientifiques de niveau recherche, publiés ou non, émanant des établissements d'enseignement et de recherche français ou étrangers, des laboratoires publics ou privés. 


\title{
The asymptotic behaviour of solutions of the forced Burgers equation on the circle
}

\author{
Patrick Bernard \\ Institut Fourier BP 74, 38402, Saint Martin d'Hères Cedex, France \\ E-mail: Patrick.Bernard@ujf-grenoble.fr
}

Received 27 March 2003, in final form 3 September 2004

Published 1 October 2004

Online at stacks.iop.org/Non/18/101

Recommended by E S Titi

\begin{abstract}
We describe the asymptotic behaviour of entropy solutions of the inviscid Burgers equation on the circle with time-periodic forcing. These solutions converge to periodic states, but the period of these limit states may be greater than the period of the forcing. A corollary is that the only solutions without shocks are periodic. This result can be interpreted in terms of the invariant curves of the associated dynamics.
\end{abstract}

Mathematics Subject Classification: 35F25, 35B40, 37E40

We study the quasi-linear first order partial differential equation

$$
\partial_{t} y+\partial_{x}(H(t, x, y))=0
$$

where $t$ is a real time, $x$ is a periodic real variable and the unknown $y$ is a real valued function of $t$ and $x$. The hypotheses on $H$ will be specified later. Equations of this kind have been extensively studied as a very simple example of turbulence. A physical model is given here, which helps the intuition. The Hamiltonian $H$ gives rise to a classical dynamics for particles on the configuration space, which is here the circle of $x$ variable. On each point $x$ of this circle, put a particle with momentum $y(0, x)$. Assume that each of these particles moves, independently of each other, driven by the classical dynamics associated with $H$. The solution $y(t, x)$ is then the momentum of the particle that is at point $x$ at time $t$. The function $y(t, x)$ may be seen as the Eulerian description of this very simple fluid. Of course, it might happen that after a certain time several different particles with different momenta have the same position. This is the reason why the equation does not have classical solutions in general. However, generalized solution can be defined by the following additional requirement: when a collision occurs between particles, these particles glue together and form what will be called a shock. Shocks then absorb the particles that hit them. Note that these shocks are not the same kind of 
objects as particles, their evolution is not driven by the Hamiltonian. The solutions describing this evolution are called the entropy solutions of $(B)$.

The main goal of this paper is to describe the asymptotic behaviour of entropy solutions of $(B)$. We prove that, if the Hamiltonian is time-periodic, each solution converges to a periodic solution. The period of the limit is a multiple of the period of the Hamiltonian. We also prove that all solutions undergo shocks, except perhaps time-periodic solutions. This does not mean, however, that periodic solutions are always free of shocks. For many Hamiltonians, there do not exist any solutions without shocks.

In order to obtain this asymptotic behaviour, we introduce another equation, satisfied by the primitive of entropy solutions, the Hamilton-Jacobi equation, whose viscosity solutions are by now well understood. In this process, we have to understand how knowledge on entropy solutions of the Burgers equation can be gained from knowledge on viscosity solutions of the associated Hamilton-Jacobi equation. Our second aim is to describe these relations with some details, and to show how most of what is known on the Burgers equation can be recovered from this point of view. As a consequence, a large part of the paper is devoted to the presentation of known results both on the Burgers equation and on the Hamilton-Jacobi equation. It is worth noticing that the trajectories of the particles, that is the Lagrangian description of our elementary fluid, play a major role in this approach.

Recently, it has been understood that the study of entropy solutions of equation $(B)$ provides interesting insights into the dynamics of the Hamiltonian $H$. This aspect is one of our motivations, and our work also implies a new observation concerning the dynamics of the time-one flow of $H$. We prove that if a curve in phase space is a graph, and is such that infinitely many of its successive images by the time-one flow are also graphs, then the curve has to be periodic, that is mapped into itself by some power of the flow. Similar results were previously obtained by Albert Fathi in the case of autonomous Hamiltonian systems.

\section{Introduction}

\section{1 .}

The standard circle $\mathbb{R} / \mathbb{Z}$ is denoted by $\mathbb{T}$. The cotangent bundle $T^{*} \mathbb{T}$ is identified with $\mathbb{T} \times \mathbb{R}$. Given a function $f(t, x)$ of two variables, we will denote by $f_{t}$ the function $x \longmapsto f(t, x)$. The partial derivative with respect to the variable $t$ will be denoted by $\partial_{t} f$. Throughout this paper, we will consider a time-periodic Hamiltonian $H(t, x, p): \mathbb{R} \times T^{*} \mathbb{T}=\mathbb{R} \times \mathbb{T} \times \mathbb{R} \longrightarrow \mathbb{R}$ and the associated time-periodic vector field of $\mathbb{T} \times \mathbb{R}$ is denoted $X$. We have

$$
X(t, x, p)=\left(\partial_{p} H(t, x, p),-\partial_{x} H(t, x, p)\right) .
$$

\section{2 .}

The following standard hypotheses will be assumed:

(i) The Hamiltonian $H$ is $C^{2}$ and 1-periodic in $t$.

(ii) The Hamiltonian $H$ is convex in $p$ and $\partial_{p p}^{2} H>0$.

(iii) The Hamiltonian has superlinear growth in $p$, i.e. $\lim _{|p| \rightarrow \infty} H(t, x, p) /|p|=\infty$ for each $(t, x)$.

(iv) The Hamiltonian flow is complete. More precisely, for all $\left(t_{0}, x_{0}, p_{0}\right)$, there exists a $C^{1}$ curve $\gamma(t)=(x(t), p(t)): \mathbb{R} \longrightarrow \mathbb{T} \times \mathbb{R}$ such that $\left(x\left(t_{0}\right), p\left(t_{0}\right)\right)=\left(x_{0}, p_{0}\right)$ and $\dot{\gamma}(t)=X(t, \gamma(t))$ for all $t \in \mathbb{R}$. 
The mapping $\gamma\left(t_{0}\right) \longmapsto \gamma(t)$ is a diffeomorphism of $\mathbb{T} \times \mathbb{R}$, denoted $\phi_{t_{0}, t}$. We will pay special attention to the diffeomorphism $\phi=\phi_{0,1}$. Note that the completeness hypothesis is satisfied if there exists a constant $C$ such that $\left|\partial_{t} H\right| \leqslant C(1+H)$.

\section{3 .}

A typical example of a Hamiltonian satisfying our hypotheses is

$$
H(t, x, p)=\frac{1}{2} p^{2}+V(t, x)
$$

with a $C^{2}$ potential $V$ periodic in $t$.

\section{4 .}

We consider the equation

$$
\partial_{t} y+\partial_{x}(H(t, x, y))=0
$$

of the unknown function $y(t, x): \mathbb{R} \times \mathbb{T} \longrightarrow \mathbb{R}$. This equation will be called the Burgers equation in the following. Note that in case $H=p^{2} / 2+V(t, x)$, we have the standard forced inviscid Burgers equation

$$
\partial_{t} y+y \partial_{x} y=-\partial_{x} V(t, x)
$$

\section{5 .}

The Burgers equation is quasi-linear and its characteristics (see [A], chapter 2) are the trajectories of $X$. This means that if $y(t, x):[a, b] \times \mathbb{T} \longrightarrow \mathbb{R}$ is a $C^{1}$ solution of the Burgers equation, and if $\gamma(t)=(x(t), p(t)):[a, b] \longrightarrow \mathbb{T} \times \mathbb{R}$ is a trajectory of the Hamiltonian vector field $X$, such that $p(a)=y(a, x(a))$, then the equality $p(t)=y(t, x(t))$ holds for all $t \in[a, b]$. In other words, for each $a \leqslant t_{0} \leqslant t_{1} \leqslant b$, the graph of the function $x \longmapsto y\left(t_{1}, x\right)$ is the image by the diffeomorphism $\phi_{t_{0}, t_{1}}$ of the graph of the function $x \longmapsto y\left(t_{0}, x\right)$.

\section{6 .}

Still assuming that $y$ is a $C^{1}$ solution of the Burgers equation, we obtain that

$$
c(t)=\int_{\mathbb{T}} y(t, x) \mathrm{d} x
$$

is a constant, which we denote $c$. The function $y$ can be written $y(t, x)=c+\partial_{x} u(t, x)$, where $u(t, x): I \times \mathbb{T} \longrightarrow \mathbb{R}$ satisfies the Hamilton-Jacobi equation

$$
\partial_{t} u+H\left(t, x, c+\partial_{x} u\right)=0 .
$$

\section{7 .}

It is known that there exists in general no classical solution of the Burgers equation defined on $\mathbb{R}^{+} \times \mathbb{T}$ satisfying a given initial condition $y(0, x)=y_{0}(x)$, see [A] or [Se]. Note that a consequence of our results is that the only classical solutions defined for all positive times are periodic in time. However, the Cauchy problem is well posed in the sense of entropy solutions (we will define entropy solutions in the following). More precisely, with all $t_{0} \in \mathbb{R}$ and all functions $y_{0} \in L^{1}(\mathbb{T})$, we associate a unique entropy solution $\left.y(t, x) \in C(] t_{0}, \infty\right), L^{1}(\mathbb{T})$ ) such that $y_{t} \longrightarrow y_{0}$ as $t \longrightarrow t_{0}$ in a weak sense, see section 3.2. This result is classical, see [Se] for example. 
1.8 .

We want to describe the asymptotic behaviour of entropy solutions. Let us first recall that for each $c$ there exists a 1-periodic solution of average $c$. More precisely, there exists a continuous and increasing function $c \longmapsto y^{c}$ from $\mathbb{R}$ to $C\left(\mathbb{R}, L^{1}(\mathbb{T})\right.$ ), which, with each $c$, associates a 1-periodic entropy solution of average $c$, see [KO] or [JKM]. Note, however, that there may exist more than one 1-periodic solution of a given average $c$. It is natural to ask whether all solutions are attracted by these 1-periodic solutions. The answer is negative; there are examples where periodic entropy solutions of minimal period greater than 1 exist, see [FM]. These subharmonic solutions in turn attract all other solutions, as we now state.

1.9 .

Theorem. Let $\left.y(t, x):] t_{0}, \infty\right) \times \mathbb{T} \longrightarrow \mathbb{R}$ be an entropy solution of the Burgers equation. There exists an integer $T$ and an entropy solution $\omega(t, x): \mathbb{R} \times \mathbb{T} \longrightarrow \mathbb{R}$ that is $T$-periodic in $t$ and such that

$$
\left\|y_{t}-\omega_{t}\right\| \longrightarrow 0
$$

in $L^{1}(\mathbb{T})$ as $t$ goes to infinity.

If $H$ is a function of $p$ only, then $\omega(t, x)$ is the constant $\int y(t, x) \mathrm{d} x$. The result in this special case has been obtained by Lax [L]. If $H$ does not depend on $t$, then the asymptotic solution $\omega$ does not depend on $t$ either, the result in this case follows from the works of Roquejoffre [Ro] and Fathi [Fa3]. The theorem will be proved in section 3 as a consequence of a similar result for viscosity solutions of $(H J c)$ obtained in [Be], see also [BR]. We will also prove the following corollary, which was obtained by Fathi [Fa3] in the autonomous case.

Corollary. If $\left.y(t, x):] t_{0}, \infty\right) \times \mathbb{T} \longrightarrow \mathbb{R}$ is a continuous solution of the Burgers equation, then the function $y$ is the restriction of a Lipschitz solution $\omega: \mathbb{R} \times \mathbb{T} \longrightarrow \mathbb{R}$ that satisfies $\omega(t+T, x)=\omega(t, x)$ for some $T \in \mathbb{N}$.

\subsection{0 .}

One can compare the situation with the viscous case. If one considers the parabolic equation

$$
\partial_{t} y+\partial_{x}(H(t, x, y))=\mu \partial_{x x} y
$$

with $\mu>0$, the behaviour is much simpler. One can prove along the lines of [JKM], see also [B], that for each $c$ there exists a unique solution $y^{c}$ of average $c$ that is 1-periodic in time. This solution attracts all the solutions of average $c$. More precisely, if $y:\left[t_{0}, \infty\right) \times \mathbb{T} \longrightarrow \mathbb{R}$ is a solution of $\left(B_{\mu}\right)$, and if $\int y_{t} \mathrm{~d} x=c$, then $y_{t}-y_{t}^{c} \longrightarrow 0$ uniformly as $t \longrightarrow \infty$.

\subsection{1 .}

The result in the inviscid case can be used to study the dynamics of the diffeomorphism $\phi=\phi_{0,1}$. Note that this diffeomorphism is a finite composition of area preserving right twist maps, and that any finite composition of right twist maps can be obtained this way. This correspondence between the twist property and the convexity of the Hamiltonian has been described by Moser, see [Mo].

\subsection{2 .}

In order to give a more geometrical meaning to theorem 1.9, we consider the set $\mathcal{E}$ of functions $f: \mathbb{T} \longrightarrow \mathbb{R}$, which can be locally written as the sum of a continuous function and a decreasing 
function. A function $f \in \mathcal{E}$ has a right limit $f^{-}(x)$ and a left limit $f^{+}(x)$ at each point $x$. These limits satisfy $f^{-}(x) \leqslant f^{+}(x)$, with a strict inequality on an at most countable set. Let $G^{-}(f)$ and $G^{+}(f)$ be the graphs in $\mathbb{T} \times \mathbb{R}$ of the functions $f^{-}$and $f^{+}$. We define the graph $G(f)=G^{-}(f) \cap G^{+}(f)$ of $f$. Note that $\overline{G(f)}=G^{-}(f) \cup G^{+}(f)$. It is also useful to consider the set

$$
H(f)=\bigcup_{x}\{x\} \times\left[f^{-}(x), f^{+}(x)\right] \subset \mathbb{T} \times \mathbb{R},
$$

which is a Jordan curve containing $\overline{G(f)}$. The Hausdorff distance $d_{H}(f, g)$ between the compact sets $H(f)$ and $H(g)$ defines a distance $d_{H}$ on $\mathcal{E}$ (one should take the quotient of $\mathcal{E}$ by the relation of almost everywhere equality). The convergence in $L^{1}$ implies convergence for the distance $d_{H}$, see section 3.3 .

\subsection{3.}

The link between entropy solutions and the dynamics of $\phi$ can now be detailed, see section 3.6. It is convenient to denote by $E: L^{1} \longrightarrow L^{1}$ the operator that associates with each initial condition $y_{0}$ the solution $y_{1}$ at time 1 . The operator $E$ is continuous. In fact, as is well known, it is a contraction. We will prove this fact in section 4. For each $y \in L^{1}$, we have $E(y) \in \mathcal{E}$. If in addition $y \in \mathcal{E}$, then

$$
\overline{G(E(y))} \subset \phi(G(y)) .
$$

This property has striking consequences. For example, if $y^{c}$ is a fixed point of $E$, then $y^{c} \in \mathcal{E}$ and $\overline{G\left(y^{c}\right)}$ is negatively invariant. As a consequence, the $\alpha$-limit of $\phi_{\mid G\left(y^{c}\right)}$ is a non-empty compact set that is fully invariant by $\phi$. It is an Aubry-Mather set. The rotation number $\rho(c) \in \mathbb{R}$ of the orbits of this set depends only on $c$ and the function $c \longmapsto \rho(c)$ is nondecreasing and continuous, see section 2.7 .

\subsection{4 .}

Having defined the rotation number $\rho(c)$ allows us to complement theorem 1.9. The asymptotic behaviour of solutions depend strongly on their space average $c$ and on the associated rotation number $\rho(c)$. If $\rho(c)$ is irrational, then there exists a single fixed point of $E$ of average $c$, as was proved in $[\mathrm{E}]$ and [So]. We will prove that it attracts all the trajectories of average $c$, that is one can take $T=1$ in theorem 1.9. If $\rho(c)$ is rational, $\rho(c)=p / q$ in lowest terms, then one can take $T=q$ in theorem 1.9. It is thus natural to define the integer $T(c)$ by $T(c)=1$ if $\rho(c)$ is irrational and $T(c)=q$ if $\rho(c)=p / q$ in lowest terms, and we have the following refinement of theorem 1.9 .

Theorem. For each entropy solution $\left.y:] t_{0}, \infty\right) \times \mathbb{T} \longrightarrow \mathbb{R}$ of $(B)$, there exists an entropy solution $\omega(t, x): \mathbb{R} \times \mathbb{T} \longrightarrow \mathbb{R}$ that is $T(c)$-periodic in time, with $c=\int_{\mathbb{T}} y_{t}(x) \mathrm{d} x$, and such that $y_{t}-\omega_{t} \longrightarrow 0$ in $L^{1}(\mathbb{T})$ as $t$ goes to infinity. As a consequence, we also have $d_{H}\left(y_{t}, \omega_{t}\right) \longrightarrow 0$.

\subsection{5 .}

Corollary 1.9 can be restated as a new result on the dynamics of $\phi$, which may be seen as a converse to the celebrated result of Birkhoff (see [Ma1, HF, Si]) stating that a rotational (not homotopic to a constant) Jordan curve in $\mathbb{T} \times \mathbb{R}$, which is invariant under $\phi$, is the graph of 
a Lipschitz function $y: \mathbb{T} \longrightarrow \mathbb{R}$. This corollary was obtained by Fathi [Fa3, Fa2] in the autonomous case.

Corollary. Let $G \subset \mathbb{T} \times \mathbb{R}$ be the graph of a continuous function $y: \mathbb{T} \longrightarrow \mathbb{R}$. Assume that there exists an increasing sequence $n_{k}$ of positive integers such that $\phi^{n_{k}}(G)$ is the graph of a continuous function. Then there exists a positive integer $T$ such that $\phi^{T}(G)=G$, and the function $y$ is Lipschitz. In addition, if $\phi(G) \neq G$, then the rotation number of $\phi_{\mid G}^{T}$ is an integer; hence, $G$ contains a $T$-periodic point of $\phi$.

Let us mention that it is certainly possible to give a more direct proof of this corollary. One could use a topological approach, as suggested to me by Le Calvez, or a variational approach, as suggested by Xia. The proof presented in section 3.9 is however extremely short.

\subsection{6 .}

In the rest of this paper, we will detail the outline given above. We will obtain all the important properties of entropy solutions of $(B)$ as consequences of properties of the viscosity solutions of $(H J c)$. Hence, we first describe these viscosity solutions in section 2 and draw our conclusions in section 3. We explain in section 4 how to understand in our framework the important fact that entropy solutions form a contraction in $L^{1}$.

\section{Calculus of variations and the Hamilton-Jacobi equation}

In this section, we describe the main properties of viscosity solutions of the equation $(H J c)$. These properties follow from the study of extremals via the Hopf-Lax-Oleinik formula; a global reference is the work of Fathi [Fa1, Fa2]. We also state a result analogous to theorem 1.9 for these solutions. The results in this section are standard or easy extensions of standard ones; however, some of the proofs are original.

\section{1.}

It is useful to introduce the Lagrangian $L: \mathbb{R} \times \mathbb{T} \times \mathbb{R} \longrightarrow \mathbb{R}$ associated with $H$. It is defined by

$$
L(t, x, v)=\sup _{p} p v-H(t, x, p)
$$

and has the following properties, which follow easily from the analogous properties 1.2 of $H$ :

(i) The Lagrangian $L$ is $C^{2}$ and 1-periodic in $t$.

(ii) The Lagrangian $L$ is convex in $v$ and $\partial_{v v}^{2} L>0$.

(iii) The Lagrangian has superlinear growth in $v$, i.e. $\lim _{|v| \rightarrow \infty} L(t, x, v) /|v|=\infty$ for each $(t, x)$.

The Lagrangian associated with the modified Hamiltonian $H(t, x, p+c)$ is $L(t, x, v)-c v$ and it satisfies the three properties given above.

\section{2.}

For each $c$ and each $t_{0} \leqslant t$, we have the Hopf-Lax-Oleinik operator $V_{t_{0}, t}^{c}: C(\mathbb{T}, \mathbb{R}) \longrightarrow$ $C(\mathbb{T}, \mathbb{R})$ defined by

$$
V_{t_{0}, t}^{c}(u)(x)=\min \left(u\left(x\left(t_{0}\right)\right)+\int_{t_{0}}^{t} L(s, x(s), \dot{x}(s))-c \dot{x}(s) \mathrm{d} s\right)
$$


where the minimum is taken on the set of absolutely continuous curves $x:\left[t_{0}, t\right] \longrightarrow \mathbb{T}$ such that $x(t)=x$. Any curve realizing the above minimum is $C^{2}$ and is the projection of a trajectory of $X$. More precisely, the curve $\left(x(s), \partial_{v} L(s, x(s), \dot{x}(s))\right)$ is a trajectory of $X$. These operators clearly satisfy the Markov property

$$
V_{t_{1}, t_{2}}^{c} \circ V_{t_{0}, t_{1}}^{c}=V_{t_{0}, t_{2}}^{c} \text {. }
$$

Note that these operators have been used in the study of viscosity solutions for quite a long time, see for instance [Fl]. We need the following definition.

Definition. A function $\tilde{u}: \mathbb{R}^{n} \longrightarrow \mathbb{R}$ is called $K$-semi-concave if the function $\tilde{u}-K\|x\|^{2} / 2$ is concave. The notion is extended to functions defined on convex subsets. A function $u:\left[t_{0}, \infty\right) \times \mathbb{T} \longrightarrow \mathbb{R}($ resp. $\mathbb{T} \longrightarrow \mathbb{R})$ is called $K$-semi-concave if it is of the form $u(t, x)=\tilde{u}(t, x \bmod 1)(\operatorname{resp} . u(x)=\tilde{u}(x \bmod 1))$, with a $K$-semi-concave function $\tilde{u}:\left[t_{0}, \infty\right) \times \mathbb{R} \longrightarrow \mathbb{R}$ (resp. $\tilde{u}: \mathbb{R} \longrightarrow \mathbb{R}$ ). A function is called semi-concave function if it can be written locally as the sum of a concave function and a $C^{2}$ function.

Properties. For each $c \in \mathbb{R}$ and each $t_{0}<t_{1}$ we have:

(i) The operators $V$ are equivariant under addition of a constant, more precisely, $V_{t_{0}, t_{1}}^{c}(U+u)=U+V_{t_{0}, t_{1}}^{c}(u)$ for all real constant $U$.

(ii) The operators $V$ are non-decreasing, the inequality $V_{t_{0}, t_{1}}^{c}(u) \leqslant V_{t_{0}, t_{1}}^{c}(v)$ holds whenever the continuous functions $u$ and $v$ satisfy $u \leqslant v$.

(iii) The operators $V$ are contractions, we have

$$
\left\|V_{t_{0}, t_{1}}^{c}(u)-V_{t_{0}, t_{1}}^{c}(v)\right\|_{\infty} \leqslant\|u-v\|_{\infty}
$$

for all continuous functions $u, v: \mathbb{T} \longrightarrow \mathbb{R}$.

(iv) For each positive $\epsilon$, there exists a constant $K$ such that, for $|c| \leqslant 1 / \epsilon$ and any continuous initial condition $u_{0}$, the function

$$
u(t, x)=V_{t_{0}, t}^{c} u_{0}(x)
$$

is $K$-Lipschitz and $K$-semi-concave on $\left[t_{0}+\epsilon, \infty\right) \times \mathbb{T}$.

2.3.

Proposition. The following properties are equivalent for a function $u \in C\left(\left[t_{0}, t_{1}\right] \times \mathbb{T}, \mathbb{R}\right)$ :

(i) The function $u$ is a viscosity solution of $(\mathrm{HJC}$ ) (see [Ba] or [Fa2] for the definition, which will not be used in this paper).

(ii) The function $u$ is locally Lipschitz on $\left.] t_{0}, t_{1}\right] \times \mathbb{T}$ and it satisfies $(H J c)$ almost everywhere. In addition, there exists a non-increasing function $\left.\left.K:] t_{0}, t_{1}\right] \longrightarrow\right] 0, \infty[$ such that the function $u_{t}$ is $K(t)$-semi-concave for $t_{0}<t \leqslant t_{1}$.

(iii) For each $t$ and $t^{\prime}$ such that $t_{0} \leqslant t \leqslant t^{\prime} \leqslant t_{1}$, we have $u_{t^{\prime}}=V_{t, t^{\prime}}^{c}\left(u_{t}\right)$.

Corollary. For each continuous initial condition $u_{t_{0}}$, there exists one and only one viscosity solution $u:\left[t_{0}, \infty\right) \times \mathbb{T} \longrightarrow \mathbb{R}$ of $(H J c)$, given by

$$
u(t, x)=V_{t_{0}, t}^{c} u_{t_{0}}(x) .
$$

There exists a non-increasing function $K(t):] t_{0}, \infty[\longrightarrow] 0, \infty[$ and such that $u$ is $K(t)$ Lipschitz and $K(t)$-semi-concave on $[t, \infty) \times \mathbb{T}$ for each $t>t_{0}$. In addition, given a constant $C>0$, the function $K(t)$ can be chosen uniformly for all equations $(H J c)$ with $|c| \leqslant C$. 
Proof. It is standard that (i) $\Longleftrightarrow$ (iii), see a good exposition in [FMa]. Let us recall a possible sketch of the proof. One can first prove using variations around the maximum principle that there is at most one function satisfying (i) with a given initial condition $u_{t_{0}}$ (see [Ba], section 2.4.). On the other hand, it is obvious that there exists one and only one function satisfying (iii), namely $(t, x) \longmapsto V_{t_{0}, t}^{c} u_{t_{0}}(x)$. One can prove, see [FMa], that this function also satisfies (i). It is then the only one to do so, by uniqueness. That (iii) $\Longrightarrow$ (ii) results from properties 2.2 . We shall prove more carefully that (ii) $\Longrightarrow$ (i), which seems less classical. Let us fix $\left.(S, Q) \in] t_{0}, \infty\right) \times \mathbb{T}$; it is enough to prove (see [Ba], section 5.3) that all $C^{1}$ functions $\phi$ such that $u-\phi$ has a local minimum at $(S, Q)$ satisfy the equation at $(S, Q)$. If such a function $\phi$ exists, then $\partial_{x} u(S, Q)$ exists and is equal to $\partial_{x} \phi(t, x)$. It follows from the lemma below that $u$ is differentiable at $(S, Q)$, and satisfies the equation at this point, which implies that $\phi$ also satisfies the equation at $(S, Q)$.

Lemma. Let $u(t, x)$ be a function satisfying condition (ii) of the proposition. If $\left.(S, Q) \in] t_{0}, \infty\right) \times \mathbb{T}$ is a point where $\partial_{x} u$ exists, then the function $u$ is differentiable and satisfies $(H J c)$ at $(S, Q)$.

Proof. Let us fix a time $\left.t_{2} \in\right] t_{0}, S$. In view of the fact that all the functions $u_{t}, t \geqslant t_{2}$ are $K\left(t_{2}\right)$-semi-concave, it is not hard to prove that $\partial_{x} u\left(s_{n}, q_{n}\right) \longrightarrow \partial_{x} u(S, Q)$ when $\left(s_{n}, q_{n}\right)$ is a sequence of points of differentiability of $u$ converging to $(S, Q)$. If we assume in addition that $(H J c)$ holds at $\left(s_{n}, q_{n}\right)$, we obtain that $\partial_{t} u\left(s_{n}, q_{n}\right)$ has a limit $H\left(t, x, c+\partial_{x} u(t, x)\right)$. Let us denote $L$ as the linear form $(s, q) \longmapsto q \partial_{x} u(S, Q)+s H\left(S, Q, \partial_{x} u(S, Q)\right)$. We have proved that there exists a modulus of continuity $\delta$ and a set $K \subset \mathbb{R} \times \mathbb{T}$ of full measure in a neighbourhood of $(S, Q)$ such that, for each $(S+s, Q+q) \in K$, the function $u$ is differentiable at $(S+s, Q+q)$ and $\|\mathrm{d} u(S+s, Q+q)-L\| \leqslant \delta(\|(s, q)\|)$. It follows that we have the estimate $|u(S+s, Q+q)-u(S, Q)-L(s, q)| \leqslant\|(s, q)\| \delta(\|(s, q)\|)$ for all $(s, q)$ small enough; hence, $u$ is differentiable at $(S, Q)$ and $\mathrm{d} u(S, Q)=L$.

2.4 .

Let $u(t, x):\left[t_{0}, \infty\right) \times \mathbb{T} \longrightarrow \mathbb{R}$ be a viscosity solution of $(H J c)$, and let $t_{0} \leqslant t<t^{\prime}$. An absolutely continuous curve $x(s):\left[t, t^{\prime}\right] \longrightarrow \mathbb{T}$ is said to be calibrated by $u$ if

$$
u\left(t^{\prime}, x\left(t^{\prime}\right)\right)=u(t, x(t))+\int_{t}^{t^{\prime}} L(s, x(s), \dot{x}(s))-c \dot{x}(s) \mathrm{d} s .
$$

If $x(s)$ is a calibrated curve, then it is $C^{2}$ and the curve $\left(x(s), \partial_{v} L(s, x(s), \dot{x}(s))\right.$ is a trajectory of the Hamiltonian vector field $X$. By extension, we say that a curve $\gamma(s)=(x(s), p(s))$ : $\left[t, t^{\prime}\right] \longrightarrow \mathbb{T} \times \mathbb{R}$ is calibrated by $u$ if $x(s)$ is calibrated by $u$ and if $p(s)=\partial_{v} L(s, x(s), \dot{x}(s))$. It is then a trajectory of $X$. A curve is said to be calibrated by $u$ on an interval $I$ if it is calibrated by $u$ on $\left[t, t^{\prime}\right]$ for all $\left[t, t^{\prime}\right] \subset I$. The following theorem is due to Albert Fathi.

\section{Theorem (Calibrated curves).}

(i) Let $\left.x(s):] t_{0}, \infty\right) \supset\left[t, t^{\prime}\right] \longrightarrow \mathbb{T}$ be a calibrated curve. For each $\left.s \in\right] t, t^{\prime}[$, the function $u$ is differentiable at $(s, x(s))$ and $\partial_{x} u(s, x(s))+c=p(s)=\partial_{v} L(s, x(s), \dot{x}(s))$.

(ii) For every $\left.(t, x) \in] t_{0}, \infty\right) \times \mathbb{T}$ there exists a calibrated curve $x(s):\left[t_{0}, t\right] \longrightarrow \mathbb{T}$ such that $x(t)=x$. If $u$ is differentiable at $(t, x)$, then there exists only one such calibrated curve and it satisfies $\partial_{x} u(t, x(t))+c=p(t)=\partial_{v} L(t, x(t), \dot{x}(t))$. 
(iii) If in addition the function $u_{t_{0}}$ is semi-concave, and if $x(s):\left[t_{0}, t\right] \longrightarrow \mathbb{T}$ is a calibrated curve, then the function $u_{t_{0}}$ is differentiable at $x\left(t_{0}\right)$ and $\partial_{x} u\left(t_{0}, x\left(t_{0}\right)\right)+c=p\left(t_{0}\right)$.

Proof. We only sketch the proof. Details can be found in [Fa2] or [Fa1]. The calibrated curve of (ii) is obtained as a minimizer of the functional

$$
u\left(t_{0}, x\left(t_{0}\right)\right)+\int_{t_{0}}^{t} L(s, x(s), \dot{x}(s))-c \dot{x}(s) \mathrm{d} s
$$

among the absolutely continuous curves $x(s):\left[t_{0}, t\right] \longrightarrow \mathbb{T}$ satisfying $x(t)=x$. The second point is Fathi's key regularity result. Let us mention the following lemma from [Fa2].

Lemma. If $\gamma(s)=(x(s), p(s)):\left[t, t^{\prime}\right] \longrightarrow \mathbb{T} \times \mathbb{R}$ is calibrated by $u$, then $p\left(t^{\prime}\right)-c$ is a proximal super-differential of $u_{t^{\prime}}$ at $x\left(t^{\prime}\right)$, i.e.

$$
u_{t^{\prime}}\left(x\left(t^{\prime}\right)+x\right) \leqslant u_{t^{\prime}}\left(x\left(t^{\prime}\right)\right)+\left(p\left(t^{\prime}\right)-c\right) x+O\left(x^{2}\right)
$$

near $x=0$, and $p(t)-c$ is a proximal sub-differential of $u_{t}$ at $x(t)$, i.e.

$$
u_{t}(x(t)+x) \geqslant u_{t}(x(t))+(p(t)-c) x+O\left(x^{2}\right) .
$$

Using this lemma, we complete the proof of the theorem. If $\gamma(s)=(x(s), p(s)):\left[t, t^{\prime}\right] \longrightarrow$ $\mathbb{T} \times \mathbb{R}$ is calibrated by $u$, and if $s \in] t, t^{\prime}\left[\right.$, then the restrictions of $\gamma$ to $[t, s]$ and $\left[s, t^{\prime}\right]$ are calibrated hence, $p(s)-c$ is both a sub-and a super-differential of $u_{s}$ at $x(s)$ and thus is the differential. This proves $(i)$. In addition, if $u_{t^{\prime}}$ is differentiable at $x\left(t^{\prime}\right)$, then its only possible sub-differential at this point is $\partial_{x} u\left(t^{\prime}, x\left(t^{\prime}\right)\right)$; hence, the calibrated curve has to satisfy the equation $\partial_{x} u\left(t^{\prime}, x\left(t^{\prime}\right)\right)+c=p\left(t^{\prime}\right)=\partial_{v} L\left(t^{\prime}, x\left(t^{\prime}\right), \dot{x}\left(t^{\prime}\right)\right)$. By Cauchy-Lipschitz uniqueness, only one characteristic can satisfy this equation. This proves the second point in (ii). The proof of (iii) is similar: the existence of a calibrated curve emanating from a point provides a sub-differential, while semi-concavity provides a super-differential.

\section{5 .}

We are interested mainly in the asymptotic behaviour of solutions. A first description is given by the following result. The function $\alpha(c)$ introduced below has been given several names, it is the $\alpha$ function of Mather, the averaged Hamiltonian, or the eigenvalue in terms of idempotent algebra.

Proposition. For each $c$, there exists a real number $\alpha(c)$ with the following property. For every solution $u(t, x):\left[t_{0}, \infty\right) \times \mathbb{T} \longrightarrow \mathbb{R}$ of $(H J c)$, the function $u(t, x)+t \alpha(c)$ is bounded on $\left[t_{0}, \infty\right) \times \mathbb{T}$.

From now on, we shall mainly consider the corrected equation

$$
\partial_{t} u+H\left(t, x, c+\partial_{x} u\right)=\alpha(c) .
$$

Proof. We work with a fixed parameter $c$. Let us define the sequences

$$
M_{n}:=\max _{x \in \mathbb{T}} V_{0, n}^{c}(0)(x) \quad \text { and } \quad m_{n}:=\min _{x \in \mathbb{T}} V_{0, n}^{c}(0)(x) .
$$

Since $V_{0, n}^{c}(0), n \geqslant 1$, are $K$-Lipschitz, we have

$$
0 \leqslant M_{n}-m_{n} \leqslant K
$$

for $n \geqslant 1$. We claim that $M_{n+m} \leqslant M_{n}+M_{m}$. This follows from the inequalities

$$
V_{0, m+n}^{c}(0)(x)=V_{0, m}^{c}\left(V_{0, n}^{c}(0)\right)(x) \leqslant V_{0, m}^{c}\left(M_{n}\right)(x) \leqslant M_{n}+V_{0, m}^{c}(0)(x) .
$$


Hence, by a classical result on subadditive sequences, we have $\lim M_{n} / n=\inf M_{n} / n$. We denote this limit by $-\alpha$. In the same way, the sequence $-m_{n}$ is subadditive; hence, $m_{n} / n \longrightarrow \sup m_{n} / n$. This limit is also $-\alpha$ because $0 \leqslant M_{n}-m_{n} \leqslant K$. As a consequence, we have, for all $n \geqslant 1$,

$$
-K-n \alpha \leqslant m_{n} \leqslant-n \alpha \leqslant M_{n} \leqslant K-n \alpha .
$$

Now let $u(t, x):\left[t_{0}, \infty\right) \times \mathbb{T} \longrightarrow \mathbb{R}$ be any solution, and let $t_{1}$ be an integer greater than $t_{0}$, then for all integers $n \in \mathbb{N}$ we obtain, for all $x$,

$\min _{\mathbb{T}} u_{t_{1}}-K \leqslant \min _{\mathbb{T}} u_{t_{1}}+m_{n}+n \alpha \leqslant u\left(n+t_{1}, x\right)+n \alpha \leqslant \max _{\mathbb{T}} u_{t_{1}}+M_{n}+n \alpha \leqslant \max _{\mathbb{T}} u_{t_{1}}+K$.

Recalling that $u$ is Lipschitz on $\left[t_{1}, \infty\right) \times \mathbb{T}$, we obtain the conclusion of the proposition with $\alpha(c)=\alpha$.

The following lemma is due to Jensen, the proof is from [BR]. The corollary is fundamental and by now classical.

Lemma. If $u(t, x):\left[t_{0}, \infty\right) \times \mathbb{T} \longrightarrow \mathbb{R}$ is a viscosity solution of $(H J c)$, the function $v(\theta, x): \mathbb{T} \times \mathbb{T} \longrightarrow \mathbb{R}$ defined by

$$
v(\theta, x)=\liminf _{t \bmod 1=\theta}(u(t, x)-t \alpha(c))
$$

is a viscosity solution of the Hamilton-Jacobi equation

$$
\partial_{\theta} v+H\left(\theta, x, c+\partial_{x} v\right)=\alpha(c),
$$

where $\theta$ denotes $t$ mod 1 .

Corollary. The Hamilton-Jacobi equation

$$
\partial_{\theta} u+H\left(\theta, x, c+\partial_{x} u\right)=a
$$

has a 1-periodic viscosity solution if and only if $a=\alpha(c)$.

Proof of the lemma. We have to prove that $V_{s, t}^{c}\left(v_{s}\right)=v_{t}$ for all $s \leqslant t$. Let us first prove that $V_{s, t}^{c}\left(v_{s}\right) \leqslant v_{t}$. In order to do so, we fix $(t, x)$ and consider an increasing sequence $n_{k}$ of integers such that $u\left(t+n_{k}, x\right) \longrightarrow v(t, x)$. There exists a sequence of curves $x_{k}:[s, t] \longrightarrow \mathbb{T}$ that is calibrated by the $u\left(t+n_{k}, x\right)$ and satisfies $x_{k}(t)=x$. We have

$$
u\left(t+n_{k}, x\right)=u\left(s+n_{k}, x_{k}(0)\right)+\int_{s}^{t} L\left(\sigma, x_{k}(\sigma+t), \dot{x}_{k}(\sigma+t)\right) \mathrm{d} \sigma .
$$

Let us assume, taking a subsequence if necessary, that the sequence $x_{k}$ is converging to $x:[s, t] \longrightarrow \mathbb{T}$ uniformly on compact sets. Taking the lim inf in the equality above gives

$$
v(t, x) \geqslant v(s, x(0))+\int_{s}^{t} L(\sigma, x(\sigma+t), \dot{x}(\sigma+t)) \mathrm{d} \sigma \geqslant V_{s, t}^{c}\left(v_{s}\right) .
$$

We assume that the functions $u_{s+n_{k}}$ have a common Lipschitz constant to conclude that $\lim \inf u\left(s+n_{k}, x_{k}(0)\right)=\lim \inf u\left(s+n_{k}, x(0)\right) \geqslant v(s, x(0))$.

In order to prove the reverse inequality, note that for all curves $x:[s, t] \longrightarrow \mathbb{T}$ we have

$$
u(t+n, x(t)) \leqslant u(s+n, x(s))+\int_{s}^{t} L(\sigma, x(\sigma), \dot{x}(\sigma)) \mathrm{d} \sigma .
$$


Taking the lim inf, we obtain

$$
v(t, x(t)) \leqslant v(s, x(s))+\int_{s}^{t} L(\sigma, x(\sigma), \dot{x}(\sigma)) \mathrm{d} \sigma
$$

for each curve $x$; hence, $V_{s, t}^{c}\left(v_{s}\right) \geqslant v_{t}$, which is the desired inequality.

2.7.

Proposition. The function $\alpha(c): \mathbb{R} \longrightarrow \mathbb{R}$ is convex, $C^{1}$, and superlinear. For each viscosity solution $u(t, x):\left[t_{0}, \infty\right) \times \mathbb{T} \longrightarrow \mathbb{R}$ of $(H J c)$, there exist curves $\gamma:\left[t_{0}, \infty\right) \longrightarrow \mathbb{T}$ that are calibrated by $u$. These curves all have the same rotation number

$$
\lim \frac{1}{t} \int_{t_{0}}^{t} \dot{x}=\rho(c):=\alpha^{\prime}(c) .
$$

Proof. We begin with superlinearity. Let $v(\theta, x)$ be a periodic solution of

$$
\partial_{\theta} v+H\left(\theta, x, c+\partial_{x} v\right)=\alpha(c) .
$$

Let $(\theta(c), x(c))$ be a minimum of $v$. The function $v$ is semi-concave and hence differentiable at $(\theta(c), x(c))$, where $\partial_{t} v=0=\partial_{x} v$. It follows that

$$
\alpha(c)=H(\theta(c), x(c), c)
$$

and the superlinearity of $\alpha$ follows from the superlinearity of $H$.

Let us continue with convexity. For fixed $t$ and $x$, the function $c \longmapsto V_{0, t}^{c}(0)(x)$ is clearly concave, since it is defined as a minimum of linear functions. It follows that the function $c \longmapsto-\alpha(c)=\lim V_{0, t}^{c}(0)(x) / t$ is concave as a limit of concave functions; hence, $\alpha(c)$ is a convex function.

Lemma. Let $u_{c}:\left[t_{0}, \infty\right) \times \mathbb{T} \longrightarrow \mathbb{R}$ be a viscosity solution of $(H J c)$ and let $x(t):\left[t_{0}, \infty\right) \longrightarrow$ $\mathbb{T}$ be calibrated by $u_{c}$, then

$$
\alpha^{\prime}(c-) \leqslant \lim \inf \frac{1}{t} \int_{t_{0}}^{t} \dot{x}(s) \mathrm{d} s \leqslant \lim \sup \frac{1}{t} \int_{t_{0}}^{t} \dot{x}(s) \mathrm{d} s \leqslant \alpha^{\prime}(c+) .
$$

Proof. See [G] for related material. Let $u_{c}:\left[t_{0}, \infty\right) \times \mathbb{T} \longrightarrow \mathbb{R}$ be a viscosity solution of $(H J c)$ and let $x(t):\left[t_{0}, \infty\right) \longrightarrow \mathbb{T}$ be calibrated by $u_{c}$. We have

$$
u_{c}(t, x(t))-u_{c}\left(t_{0}, x\left(t_{0}\right)\right)=\int_{t_{0}}^{t} L(s, x(s), \dot{x}(s)) \mathrm{d} s-c \int_{t_{0}}^{t} \dot{x}(s) \mathrm{d} s .
$$

In view of the definition of $\alpha(c)$ in section 2.5, the function $u_{c}(t, x)+t \alpha(c)$ is bounded; hence,

$$
\lim _{t \rightarrow \infty}\left(\frac{c}{t} \int_{t_{0}}^{t} \dot{x}(s) \mathrm{d} s-\frac{1}{t} \int_{t_{0}}^{t} L(s, x(s), \dot{x}(s)) \mathrm{d} s\right)=\alpha(c) .
$$

Let us now consider a viscosity solution $u_{e}:\left[t_{0}, \infty\right) \times \mathbb{T} \longrightarrow \mathbb{R}$ of $($ HJe $)$. With the same curve $x(s)$, we have

$$
u_{e}(t, x(t))-u_{e}\left(t_{0}, x\left(t_{0}\right)\right) \leqslant \int_{t_{0}}^{t} L(s, x(s), \dot{x}(s)) \mathrm{d} s-e \int_{t_{0}}^{t} \dot{x}(s) \mathrm{d} s
$$

hence,

$$
\alpha(e) \geqslant \lim \sup \left(\frac{e}{t} \int_{t_{0}}^{t} \dot{x}(s) \mathrm{d} s-\frac{1}{t} \int_{t_{0}}^{t} L(s, x(s), \dot{x}(s)) \mathrm{d} s\right),
$$


which implies

$$
\alpha(e)-\alpha(c) \geqslant \lim \sup \left(\frac{e-c}{t} \int_{t_{0}}^{t} \dot{x}(s) \mathrm{d} s\right) .
$$

The desired inequalities follow at the limit.

We still have to prove that the function $\alpha$ is differentiable. We need some preliminaries.

\section{8 .}

Let $\mathcal{C}$ be the set of continuous curves $x(t): \mathbb{R} \longrightarrow \mathbb{T}$ such that the mapping $t \longmapsto \tilde{x}(t)=$ $(t \bmod 1, x(t))$ with values in $\mathbb{T}^{2}$ is either one to one or periodic. Let us endow $\mathcal{C}$ with the topology of uniform convergence on compact sets. The following result of Poincaré is very standard and will not be proved here.

Proposition. Each curve $x \in \mathcal{C}$ has a well defined real rotation number

$$
\rho(x):=\lim \frac{1}{t} \int_{0}^{t} \dot{x}(s) \mathrm{d} s .
$$

In addition, the mapping $x \longmapsto \rho(x)$ is continuous on $\mathcal{C}$. If $x(t)$ and $y(t)$ are two curves of $\mathcal{C}$ such that $\tilde{x}(\mathbb{R})$ and $\tilde{q}(\mathbb{R})$ are disjoint subsets of $\mathbb{T}^{2}$, then they have the same rotation number.

\section{9.}

Lemma. Let $u(\theta, x)$ and $v(\theta, x)$ be two viscosity solutions of $(H c)$ of time period 1 . Then there exists a curve $x(t): \mathbb{R} \longrightarrow \mathbb{T}$ that is calibrated both by $u$ and $v$.

It is usual in Aubry-Mather theory to define the Aubry set as the unions of all curves that are calibrated by all 1-periodic viscosity solutions of $(\mathrm{Hc})$. It is known that the Aubry set is not empty. This result of course implies the lemma, but we provide a simple self-contained proof.

Proof. Let us define the number $M(t):=\max \left(v_{t}-u_{t}\right)$. The function $M(t)$ is non-increasing, because the inequality $v_{t} \leqslant M+u_{t}$ implies $v_{s} \leqslant M+u_{s}$ for all $s \geqslant t$, by properties 2.2(i) and (ii). On the other hand, the functions $u$ and $v$ are periodic; hence, $M(t)$ is periodic and constant. We denote this constant by $M$. Let us fix a time $t$ and a point $x_{t}$ such that $v\left(t, x_{t}\right)=M(t)+u\left(t, x_{t}\right)$. Let $x(s):(-\infty, 0] \longrightarrow \mathbb{T}$ be a curve calibrated by $u$ and such $x(t)=x_{t}$. Since

$$
u(t, x(t))=u(s, x(s))+\int_{s}^{t} L(s, x(s), \dot{x}(s))-c \dot{x}(s) \mathrm{d} s
$$

and

$$
v(t, x(t)) \leqslant v(s, x(s))+\int_{s}^{t} L(s, x(s), \dot{x}(s))-c \dot{x}(s) \mathrm{d} s
$$

for all $s<t \leqslant 0$, we obtain

$$
M \geqslant v(s, x(s))-u(s, x(s)) \geqslant v(t, x(t))-u(t, x(t)) \geqslant M
$$

hence, the inequalities are all equalities, which imply that the curve $x(s)$ is also calibrated by $v$. We have found a curve $x(s):(-\infty, 0] \longrightarrow \mathbb{T}$ that is calibrated by $u$ and $v$. We now consider a curve $\bar{x}(s): \mathbb{R} \longrightarrow \mathbb{T}$ obtained as an accumulation point of the sequence $x(s-n), n \in \mathbb{N}$. This curve is calibrated by $u$ and $v$. 
Corollary. There exists a real number $\rho(c) \in\left[\alpha^{\prime}(c-), \alpha^{\prime}(c+)\right]$ such that each curve $x: \mathbb{R} \longrightarrow \mathbb{T}$ calibrated by a 1-periodic viscosity solution $u(\theta, x)$ of $(H J c)$ has rotation number $\rho(c)$.

Proof. It is easy to see that, if $x$ is calibrated by a periodic solution, then $x \in \mathcal{C}$; hence, $x$ has a rotation number. Let us consider two curves $x(t)$ and $q(t)$, such that $x$ is calibrated by the 1-periodic solution $u(\theta, x)$ of $(H c)$ and $q$ is calibrated by the 1-periodic solution $v(\theta, x)$ of $(H c)$. In view of the lemma, there exists a third curve $f(t)$ that is calibrated both by $u$ and $v$. Since $x$ and $f$ are both calibrated by $u$, the sets $\tilde{x}(\mathbb{R})$ and $\tilde{f}(\mathbb{R})$ are either disjoint or equal. In each case, we have $\rho(x)=\rho(f)$. In the same way, $f$ and $q$ are both calibrated by $v$; hence, $\rho(q)=\rho(f)$. As a consequence, we have $\rho(q)=\rho(x)$. Hence, all the curves that are calibrated by some 1-periodic solution of $(H c)$ have the same rotation number and we can denote this rotation number by $\rho(c)$. It follows from lemma 2.7 that $\rho(c) \in\left[\alpha^{\prime}(c-), \alpha^{\prime}(c+)\right]$.

\subsection{0 .}

We are going to prove that the function $\rho(c)$ is continuous. This implies the differentiability of $\alpha$ and completes the proof of proposition 2.7. Let us fix a value of $c$ and consider a sequence $c_{n}$ converging to $c$. Let $v_{n}(\theta, x)$ be a time-periodic viscosity solution of $\left(H c_{n}\right)$ and let $x_{n}(t): \mathbb{R} \longrightarrow \mathbb{R}$ be a curve calibrated by $v_{n}$. We extract a subsequence $k$ of $n$ in such a way that the sequence $v_{k}$ converges, uniformly on compact subsets of $\mathbb{R} \times \mathbb{T}$, to a viscosity solution $v$ of the equation $(H c)$. In addition, we can suppose that the curves $x_{k}$ are converging uniformly on compact sets to a curve $x(t)$ that is calibrated by $v$. By the definition of $\rho(c)$, we have $\rho\left(x_{k}\right)=\rho\left(c_{k}\right)$ and $\rho(x)=\rho(c)$. By continuity of the rotation number on $\mathcal{C}$, we have $\rho\left(x_{k}\right) \longrightarrow \rho(x)$; hence, $\rho\left(c_{k}\right) \longrightarrow \rho(c)$. This is the expected continuity of $\rho$. We have proved proposition 2.7 .

\subsection{1 .}

The asymptotic behaviour of viscosity solutions is described by the following theorem, obtained in [Be] (see also [BR] for another proof, and see [Fa3] and [Ro] for related results). Let $T(c) \in \mathbb{N}$ be defined by

$$
\begin{aligned}
& T(c)=1 \text { if } \rho(c) \text { is irrational, } \\
& T(c)=q \text { if } \rho(c) \text { is the rational } p / q \text { in lowest terms. }
\end{aligned}
$$

Theorem. Let $u(t, x):\left[t_{0}, \infty\right) \times \mathbb{T} \longrightarrow \mathbb{R}$ be a viscosity solution of $(H J c)$. There exists a viscosity solution $v(t, x): \mathbb{R} \times \mathbb{T} \longrightarrow \mathbb{R}$ of $(H J c)$ that satisfies $v_{t+T(c)}=v_{t}-T(c) \alpha(c)$ for each $t \in \mathbb{R}$ and

$$
\lim _{t \rightarrow \infty}\left\|u_{t}-v_{t}\right\|_{\infty}=0
$$

\section{Entropy solutions and characteristics}

The relation between classical solutions of Burgers equation and the Hamiltonian dynamics is quite well understood from section 1.5. We shall now describe the main properties of entropy solutions, with emphasis on their relation with dynamics. We will also prove theorem 1.14 and corollary 1.15 . 
3.1 .

A function $y:] t_{0}, t_{1}[\longrightarrow \mathbb{R}$ is called an entropy solution of Burgers equation $(B)$ if:

(i) The functions $y$ and $H(t, x, y(t, x))$ are locally integrable and the equation holds in the sense of distributions:

$$
\int_{\left[t_{0}, \infty\right) \times \mathbb{T}} y(t, x) \partial_{t} \phi(t, x)+H(t, x, y(t, x)) \partial_{x} \phi(t, x) \mathrm{d} t \mathrm{~d} x=0
$$

for all smooth function $\phi:] t_{0}, t_{1}[\times \mathbb{T} \longrightarrow \mathbb{R}$ with compact support (see [Se] for details). Note that the space average $\int_{\mathbb{T}} y(t, x) \mathrm{d} x$ is then a constant $c$.

(ii) The Oleinik inequalities

$$
y_{t}(x+\delta)-y_{t}(x) \leqslant K(t) \delta
$$

hold for all $t \in] t_{0}, t_{1}[, x \in \mathbb{T}$ and $\delta>0$, with a positive and decreasing function $K(t)$.

\section{2 .}

Let $y_{n}$ be a sequence of functions of $L^{1}(\mathbb{T})$. Considering these functions as periodic functions on $\mathbb{R}$, we can define the primitives $Y_{n}(x):=\int_{0}^{x} y_{n}$. We say that the sequence $y_{n}$ converges very weakly to $y$ if the primitives $Y_{n}$ converge to $Y$, uniformly on compact sets.

Proposition. For each $t_{0} \in \mathbb{R}$ and each $y_{0} \in L^{1}(\mathbb{T})$, there exists a unique entropy solution $\left.y:] t_{0}, \infty\right) \times \mathbb{T} \longrightarrow \mathbb{R}$ of Burgers equation such that $y_{t}$ converges very weakly ${ }^{1}$ to $y_{0}$ when $t$ converges to $t_{0}$. This solution satisfies

$$
\left.\left.y \in L^{\infty}\left(\left[t_{1}, \infty\right) \times \mathbb{T}\right) \cap C(] t_{0}, \infty\right), L^{1}(\mathbb{T})\right)
$$

for all $t_{1}>t_{0}$, and it is given by $y(t, x)=c+\partial_{x} u(t, x)$, where $c=\int_{\mathbb{T}} y_{0} \mathrm{~d} x$ and $u(t, x)$ is the viscosity solution of $(H J c)$ of initial condition $u_{t_{0}}(x)=\int_{0}^{x}\left(y_{0}-c\right)$.

Proof. Let us first deal with uniqueness. The standard method to prove uniqueness is to use the Oleinik inequalities 3.1(ii), via a duality method; see [H], theorem 2.2.1, or [Se], 2.8. We shall use the Hamilton-Jacobi equation. Indeed, let $\left.y(t, x):] t_{0}, \infty\right) \times \mathbb{T} \longrightarrow \mathbb{R}$ be an entropy solution. Note that this function is locally bounded in view of the Oleinik inequalities. Define

$$
\tilde{u}(t, x)=\int_{0}^{x}(y(t, q)-c) \mathrm{d} q-\int_{0}^{1} \int_{0}^{x}(y(t, q)-c) \mathrm{d} q \mathrm{~d} x,
$$

where $c=\int_{\mathbb{T}} y_{t} \mathrm{~d} x$. We have, in the sense of distributions, $\partial_{t x} \tilde{u}=\partial_{t} y=-\partial_{x}(H(t, x, y))$. Hence, the distribution $\partial_{t} \tilde{u}(t, x)+H(t, x, y(t, x))$ does not depend on $x$ and is the locally integrable function $f(t)=\int_{\mathbb{T}} H(t, x, y(t, x)) \mathrm{d} x$. The function $u(t, x)=\tilde{u}(t, x)-\int_{t_{0}}^{t} f(s) \mathrm{d} s$ satisfies $\partial_{t} u+H(t, x, y)=0$ and $\partial_{x} u=y-c$ in the sense of distributions; hence, both $\partial_{x} u$ and $\partial_{t} u$ are locally bounded functions on $\left.] t_{0}, \infty\right) \times \mathbb{T}$ and (see, e.g., [EG], section 4.2.3) the function $u$ is locally Lipschitz on $\left.] t_{0}, \infty\right) \times \mathbb{T}$. In addition, the Hamilton-Jacobi equation $\partial_{t} u+H\left(t, x, c+\partial_{x} u\right)=0$ holds almost everywhere. The function $u$ satisfies condition (ii) of proposition 2.3 as a consequence of the Oleinik inequalities (ii) above. Since the functions $y_{t}$ converge very weakly to $y_{0}$ as $t \longrightarrow t_{0}$, we have $\tilde{u}_{t} \longrightarrow u_{t_{0}}$ uniformly as $t \longrightarrow t_{0}$, which implies that $u_{t} \longrightarrow u_{t_{0}}$ uniformly. As a consequence, the function $u$ is continuous on $\left[t_{0}, \infty\right) \times \mathbb{T}$. In view of section 2.3 , the function $u$ has to be the viscosity solution of $(H J c)$ of initial condition $u_{t_{0}}$. So the only candidate to be an entropy solution of $(B)$ is $y(t, x)=c+\partial_{x} u(t, x)$.

1 The nature of the convergence of entropy solutions to their initial condition can be described more precisely depending on the regularity of the initial condition. This question, however, is not very relevant to our discussion. 
It is classical to obtain the existence of entropy solutions as limits of regular solutions of the viscous equation $\left(B_{\mu}\right)$. However, we shall use once more the Hamilton-Jacobi equation, i.e. we shall prove that the function $y(t, x)=c+\partial_{x} u(t, x)$ introduced in the discussion on uniqueness is indeed an entropy solution. Recall that $u$ is Lipschitz on $\left[t_{1}, \infty\right) \times \mathbb{T}$ for all $t_{1} \geqslant t_{0}$; hence, $\partial_{t} u$ and $y=c+\partial_{x} u$ are well defined in $L^{\infty}\left(\left[t_{1}, \infty\right) \times \mathbb{T}\right)$ as well as in $H(t, x, y(t, x))$. It is straightforward that (i) is satisfied and (ii) follows from property (ii) of proposition 2.3.

\section{3 .}

In order to prove the continuity of the mapping $t \longmapsto y_{t}$ from $\left.] t_{0}, \infty\right)$ to $L^{1}(\mathbb{T})$, let us introduce, for each positive number $K$, the set $\mathcal{E}_{K}$ of functions that satisfy both

$$
|y(x)| \leqslant K \quad \text { for all } x
$$

and

$$
y(x+\delta) \leqslant K \delta+y(x) \quad \text { for all } x \in \mathbb{T} \quad \text { and } \quad \delta>0
$$

Lemma. The set $\mathcal{E}_{K}$ is relatively compact in $L^{1}(\mathbb{T})$ (for the strong topology). In addition, there exists a modulus of continuity $C_{K}(\epsilon)$ such that

$$
d_{H}(y, z) \leqslant C_{K}\left(\|y-z\|_{L^{1}}\right)
$$

for $y, z \in \mathcal{E}_{K}$, where $d_{H}$ is the distance defined in section 1.12.

Proof. Let us denote by $\tau_{\delta} y$ the function $x \longmapsto y(x+\delta)$. We claim that, for each $\delta \in \mathbb{R}$, we have $\left\|\tau_{\delta} y-y\right\|_{L^{1}} \leqslant 2 K|\delta|$. In view of the Riesz-Fréchet-Kolmogorov compactness criterion (see [Br], IV.5) this implies the first part of the lemma. In order to prove the claim, let us first suppose that $\delta>0$. Using the Oleinik inequality, we get $\tau_{\delta} y-y \leqslant K \delta$. Let us set $z^{+}(x)=\max \left\{0, \tau_{\delta} y(x)-y(x)\right\}$ and $z^{-}=\max \left\{0, y(x)-\tau_{\delta} y(x)\right\}$, so that $\tau_{\delta} y-y=z^{+}-z^{-}$. We have $z^{+} \leqslant K \delta$; hence, $\int_{\mathbb{T}} z^{+} \mathrm{d} x \leqslant K \delta$. Noticing that $\int_{\mathbb{T}}\left(\tau_{\delta} y-y\right) \mathrm{d} x=0$, we obtain that $\int_{\mathbb{T}} z^{-} \mathrm{d} x=\int_{\mathbb{T}} z^{+} \mathrm{d} x ;$ hence,

$$
\left\|\tau_{\delta} y-y\right\|_{L^{1}}=\int_{\mathbb{T}} z^{+} \mathrm{d} x+\int_{\mathbb{T}} z^{-} \mathrm{d} x \leqslant 2 K \delta .
$$

The inequality for $\delta<0$ follows in the same way from the fact that $\tau_{\delta} y-y \geqslant K \delta$ in this case.

We now prove the second part of the lemma. See [KO] for related material. Let us consider two functions $y$ and $z$ of $\mathcal{E}_{K}$ and assume that $d_{H}(y, z) \geqslant 2 \epsilon>0$. There exists a point $\left(x_{0}, y_{0}\right) \in H(y)$ (see section 1.12 for the notation) such that the ball $B$ of radius $2 \epsilon$ centred at $\left(x_{0}, y_{0}\right)$ in $\mathbb{T} \times \mathbb{R}$ does not contain any point of $H(z)$. Hence, either $z(x) \geqslant y_{0}+\epsilon$ for almost all $x \in\left[x_{0}-\epsilon, x_{0}+\epsilon\right]$ or $z(x) \leqslant y\left(x_{0}\right)-\epsilon$ for almost all $x \in\left[x_{0}-\epsilon, x_{0}+\epsilon\right]$. We will treat the first case, the second being similar. Using the Oleinik inequality, we obtain, for each $\delta \in[0, \epsilon]$,

$$
z\left(x_{0}+\delta\right) \geqslant y_{0}+\epsilon \geqslant y\left(x_{0}+\delta\right)-K \delta+\epsilon .
$$

Hence,

$$
\|y-z\|_{L^{1}} \geqslant \int_{x_{0}}^{x_{0}+\min \{\epsilon, \epsilon / K\}} z(x)-y(x) \mathrm{d} x \geqslant \int_{0}^{\min \{\epsilon, \epsilon / K\}} \epsilon-K x \mathrm{~d} x \geqslant \epsilon^{2} \max \left(\frac{1}{2}, \frac{1}{2 K}\right) .
$$

Corollary. Let $\mathcal{V}_{K} \subset C(\mathbb{T}, \mathbb{R})$ be the set of $K$-semi-concave (hence $K$-Lipschitz) functions. Then every function $u \in \mathcal{V}_{K}$ has a derivative $\partial_{x} u$ in $\mathcal{E}_{K}$, and the operator $\partial_{x}: \mathcal{V}_{K} \rightarrow \mathcal{E}_{K}$ is 
uniformly continuous, when $\mathcal{V}_{K}$ is endowed with the topology of uniform convergence and $\mathcal{E}_{K}$ with the strong $L^{1}$ topology.

Proof. In order to prove that the operator $\partial_{x}: \mathcal{V}_{K} \rightarrow \mathcal{E}_{K}$ is uniformly continuous, let us consider two sequences $u_{n}$ and $v_{n}$ of $\mathcal{V}_{K}$, such that $\left\|u_{n}-v_{n}\right\|_{\infty} \longrightarrow 0$. We have to prove that $\left\|\partial_{x} u_{n}-\partial_{x} v_{n}\right\|_{L^{1}} \longrightarrow 0$. If not, there would exist subsequences $u_{k}$ and $v_{k}$ such that $\partial_{x} u_{k}$ and $\partial_{x} v_{k}$ have different limits $y$ and $z$ in $L^{1}$. This implies that the sequences $u_{k}$ and $v_{k}$ have limits $u$ and $v$ in $C(M, \mathbb{R})$, and that these limits are different. But this is obviously in contradiction with the assumption that $\left\|u_{n}-v_{n}\right\|_{\infty} \longrightarrow 0$.

In view of this corollary, the continuity of $t \longmapsto y_{t}$ follows from the continuity of $t \longmapsto u_{t}$.

\section{4 .}

It is clear from what has just been written that theorem 2.11 implies our main new results, theorems 1.9 and 1.14. Indeed, if $y(t, x)$ is an entropy solution of $(B)$, we write it $y=c+\partial_{x} u$, where $u$ is a viscosity solution of $(H J c)$. Theorem 2.11 gives the existence of a viscosity solution $v$ of $(H J c)$ such that $v(t+T(c), x)=v(t, x)-T(c) \alpha(c)$ and $\left\|v_{t}-u_{t}\right\|_{\infty} \longrightarrow 0$ as $t \longrightarrow \infty$. It follows from the considerations above that the function $\omega(t, x)=c+\partial_{x} v(t, x)$ is an entropy solution of $(B)$, and it clearly satisfies $\omega(t+T(c), x)=\omega(t, x)$. In addition, for $t_{1}>t_{0}$, all the functions $u_{t}, t \geqslant t_{1}$ and $v_{t}, t \in \mathbb{R}$ belong to the same set $\mathcal{V}_{K}$. We obtain that $\left\|y_{t}-\omega_{t}\right\|_{L^{1}}=\left\|\partial_{x} u_{t}-\partial_{x} v_{t}\right\|_{L^{1}} \longrightarrow 0$ as $t \longrightarrow \infty$ in view of the uniform continuity of the operator $\partial_{x}: \mathcal{V}_{K} \rightarrow \mathcal{E}_{K}$. The second part of lemma 3.3 then implies that $d_{H}\left(y_{t}, \omega_{t}\right) \longrightarrow 0$.

\section{5 .}

Consider an entropy solution $y(t, x):\left[t_{0}, \infty\right) \times \mathbb{T} \longrightarrow \mathbb{R}$ of Burgers equation, let $c=\int_{\mathbb{T}} y \mathrm{~d} x$ and let $u(t, x):\left[t_{0}, \infty\right) \times \mathbb{T} \longrightarrow \mathbb{R}$ be a viscosity solution of $(H J c)$ such that $y=c+\partial_{x} u$. A trajectory $\gamma(s):\left[t_{0}, \infty\right) \supset\left[t, t^{\prime}\right] \longrightarrow \mathbb{T} \times \mathbb{R}$ of the Hamiltonian vector field $X$ is called a $y$-characteristic if $\gamma(s) \in \overline{G\left(y_{s}\right)}$ for each $s \in\left[t, t^{\prime}\right]$. A curve $x(s):\left[t_{0}, \infty\right) \supset\left[t, t^{\prime}\right] \longrightarrow \mathbb{T}$ is also called a $y$-characteristic if it is the projection of a $y$-characteristic $\gamma(s)$. The following theorem extends the method of characteristics to entropy solutions.

\section{Theorem (characteristics).}

(i) A curve $\left.\gamma(s):] t_{0}, \infty\right) \supset\left[t, t^{\prime}\right] \longrightarrow \mathbb{T} \times \mathbb{R}$ is a y-characteristic if and only if it is calibrated by $u$.

(ii) Let $\left.\gamma(s):] t_{0}, \infty\right) \supset\left[t, t^{\prime}\right] \longrightarrow \mathbb{T} \times \mathbb{R}$ be a $y$-characteristic, then for each $\left.s \in\right] t, t^{\prime}[$ the function $y_{s}$ is continuous at $x(s)$ and $y(s, x(s))=p(s)$.

(iii) For every $t>t_{0}$ and every $(x, p) \in \overline{G\left(y_{t}\right)}$ there exists a unique $y$-characteristic $\left.\gamma(s):] t_{0}, t\right] \longrightarrow \mathbb{T} \times \mathbb{R}$ such that $\gamma(t)=(x, p)$.

(iv) If in addition $y_{t_{0}} \in \mathcal{E}$, then for every $t>t_{0}$ and every characteristic $\left.x:\right] t_{0}, t[\longmapsto \mathbb{T}$ we have $p\left(t_{0}\right)=y\left(t_{0}, x\left(t_{0}\right)\right)$, where $\left(x\left(t_{0}\right), p\left(t_{0}\right)\right)$ is naturally defined by prolongation of the Hamiltonian trajectory $(x(t), p(t))$.

(v) There exists a characteristic $x(t):\left[t_{0}, \infty\right) \longrightarrow \mathbb{T}$.

Proof. The proposition will be deduced from the properties of calibrated curves mentioned in section 2.4. If $\gamma(s)=(x(s), p(s)):\left[t, t^{\prime}\right] \longrightarrow \mathbb{T} \times \mathbb{R}$ is a calibrated curve, then we have 
seen that $y$ is continuous at $(s, x(s))$ for each $s$ of $] t, t^{\prime}[$ and that $y(s, x(s))=p(s)$. As a consequence, calibrated curves are characteristics and (ii) holds for these curves.

Let us now prove (iii). Let us fix a point $(x, p) \in \overline{G\left(y_{t}\right)}$ and let $\left(x_{n}, p_{n}\right)$ be a sequence of points of $G\left(y_{t}\right)$ converging to $(x, p)$. For each $n$, there exists a calibrated curve $\left(x_{n}(s), p_{n}(s)\right):\left[t_{0}, t\right] \longrightarrow \mathbb{T} \times \mathbb{R}$ such that $x_{n}(t)=x_{n}$. Since $\left(t, x_{n}\right)$ is a point of differentiability of $u$, this calibrated curve satisfies $p_{n}(t)=\partial_{x} u\left(t, x_{n}\right)+c=p_{n}$. These calibrated curves converge to a calibrated curve that terminates at $(t, x, p)$. Uniqueness in (iii) is a consequence of the Cauchy-Lipschitz theorem for the Hamiltonian vector field.

We now prove that all characteristics are calibrated curves. Let $\gamma(s)=(x(s), p(s))$ : $\left[t, t^{\prime}\right] \longrightarrow \mathbb{T} \times \mathbb{R}$ be a characteristic. Let $\tilde{\gamma}(s):\left[t_{0}, t^{\prime}\right] \longrightarrow \mathbb{T} \times \mathbb{R}$ be the unique characteristic terminating at $\left(t^{\prime}, x\left(t^{\prime}\right), p\left(t^{\prime}\right)\right)$, which was obtained above (and which is a calibrated curve). Clearly, $\gamma$ has to be the restriction of $\tilde{\gamma}$, hence it is a calibrated curve.

\section{6 .}

In terms of the dynamics, using the notation of section 1.12, this theorem implies that

$$
\overline{G\left(y_{t^{\prime}}\right)} \subset \phi_{t, t^{\prime}}\left(G\left(y_{t}\right)\right)
$$

when $t_{0}<t<t^{\prime}$, and that

$$
\overline{G(E(y))} \subset \phi(G(y))
$$

when $y \in \mathcal{E}$.

\section{7 .}

A function $y:] t_{0}, t_{1}[\times \mathbb{T} \longrightarrow \mathbb{R}$ is called a backward entropy solution of $(B)$ if the function $\breve{y}(s, x):=-y\left(t_{1}-s\right)$ is an entropy solution of the reverse equation

$$
\partial_{t} \breve{y}+\partial_{x}(\breve{H}(t, x, y))=0,
$$

where $\breve{H}(t, x, p)=H\left(t_{1}-t, x,-p\right)$. If $y$ is a $C^{1}$ solution of the Burgers equation, then it is an entropy solution and a backward entropy solution. However, entropy solutions and backward entropy solutions are different in general. More precisely, the function $y$ is a backward entropy solution if and only if it is a solution in the sense of distributions, and satisfies the reversed Oleinik inequalities

$$
y_{t}(x+\delta) \geqslant y_{t}(x)-K(t) \delta
$$

when $\delta>0$, with an increasing function $\left.K:] t_{0}, t_{1}[\longrightarrow] 0, \infty\right)$. As a consequence, if $y$ is both an entropy solution and a backward entropy solution, then $y_{t}$ is Lipschitz for each $t \in] t_{0}, t_{1}[$. More precisely, we have the following proposition.

Proposition. Let $\left.y:] t_{0}, \infty\right) \times \mathbb{T} \longrightarrow \mathbb{R}$ be an entropy solution of $(B)$. The function $y$ is a backward entropy solution of $(B)$ on $] t_{0}, t_{1}\left[\times \mathbb{T}\right.$ if and only if the function $y_{t_{1}}$ is continuous. The function $y$ is then locally Lipschitz on $] t_{0}, t_{1}[\times \mathbb{T}$.

Proof. Let us first assume that $y$ is both an entropy solution and a backward entropy solution. Then for each compact interval of time $I \subset] t_{0}, t_{1}\left[\right.$, the functions $y_{t}, t \in I$ are equi-Lipschitz. In view of the lemma in section 2.3, we conclude that $y$ is Lipschitz on $I$.

Assume now that $y_{t_{1}}$ is continuous. Then each point $\left(x, y_{t_{1}}(x)\right)$ is the endpoint of a single characteristic $\left.\left.\Gamma_{x}(t)=(Q(t, x), P(t, x)):\right] t_{0}, t_{1}\right] \longrightarrow \mathbb{T}$. Clearly, we have $y(t, Q(t, x))=$ $P(t, x)$ for all $\left.(t, x) \in] t_{0}, t_{1}\right] \times \mathbb{T}$. Let $z(t, x):\left(-\infty, t_{1}\right] \times \mathbb{T} \longrightarrow \mathbb{R}$ be the unique backward entropy solution with final condition $y_{t_{1}}$ given by proposition 3.2. Let us fix a point 
$(t, x) \in\left[t_{0}, t_{1}\right] \times \mathbb{T}$ and a $z$-characteristic $\gamma(s)=(x(s), p(s)):\left[t, t_{1}\right] \longrightarrow \mathbb{T} \times \mathbb{R}$ satisfying $x(t)=x$. Since $-y_{t_{1}} \in \mathcal{E}$, this characteristic furthermore satisfies $p\left(t_{1}\right)=z\left(t_{1}, x\left(t_{1}\right)\right)$ (this results from (iv) of the theorem on characteristics). As a consequence, the characteristic $\gamma(s)$ coincides with $\Gamma_{x\left(t_{1}\right)}(s)$. This implies that $z_{t}$ is continuous at $x$ and satisfies

$$
z(t, x)=p(t)=P\left(t, x\left(t_{1}\right)\right)=y\left(t, Q\left(t, x\left(t_{1}\right)\right)\right)=y(t, x) .
$$

We have proved that $y_{t}=z_{t}$ for $\left.t \in\right] t_{0}, t_{1}[$.

3.8 .

Proposition. Let $\left.y, z:] t_{0}, \infty\right) \times \mathbb{T} \longrightarrow \mathbb{R}$ be two continuous entropy solutions of $(B)$. Then the function $t \longmapsto\left\|y_{t}-z_{t}\right\|_{L^{1}}$ is constant.

This result will be extended in section 4, where we give a proof of the well known fact that the function $t \longmapsto\left\|y_{t}-z_{t}\right\|_{L^{1}}$ is non-increasing if $y$ and $z$ are entropy solutions. Since continuous solutions are both entropy solutions and backward entropy solutions, this implies the proposition. However, we give an independent proof here.

Proof. Let us consider the graphs $\Gamma_{y_{t}}$ and $\Gamma_{z_{t}}$ of $y$ and $z$ in $\mathbb{T} \times \mathbb{R}$. Let $U_{t}$ be the domain enclosed by these two curves, that is the union of all bounded connected components of $\mathbb{T} \times \mathbb{R}-\left(\Gamma_{y_{t}} \cup \Gamma_{z_{t}}\right)$. Clearly, $\left\|y_{t}-z_{t}\right\|_{L^{1}}$ is the area of $U_{t}$. It follows from the theorem on characteristics that $U_{t}^{\prime}=\phi_{t, t^{\prime}}\left(U_{t}\right)$ for $t^{\prime}>t>t_{0}$, where $\phi$ is the Hamiltonian flow. The area of $U_{t}$ does not depend on $t$ because the flow is area preserving.

\section{9 .}

We are now in a position to prove corollary 1.9. Let us first state it in slightly greater generality.

Corollary. Let $\left.y(t, x):] t_{0}, \infty\right) \times \mathbb{T} \longrightarrow \mathbb{R}$ be a solution of the Burgers equation. If there exists an unbounded sequence $t_{n}>t_{0}$ of times such that the function $y_{t_{n}}$ is continuous for all $n$, then the function $y$ is the restriction of a Lipschitz solution $\omega: \mathbb{R} \times \mathbb{T} \longrightarrow \mathbb{R}$ that satisfies $\omega(t+T, x)=\omega(t, x)$ for some $T \in \mathbb{N}$.

Proof. It follows from proposition 3.7 that the function $y$ is both a forward entropy solution and a backward entropy solution on $] t_{0}, t_{n}[\times \mathbb{T}$ for each $n$. It follows from the Oleinik inequality and the reversed Oleinik inequality that the function $y$ is Lipschitz on $\left[t_{0}^{\prime}, \infty\right) \times \mathbb{T}$ for all $t_{0}^{\prime}>t_{0}$. On the other hand, there exists a periodic solution $\omega(t, x)$ such that $\left\|y_{t}-\omega_{t}\right\|_{L^{1}} \longrightarrow 0$ as $t \longrightarrow \infty$. The functions $\omega_{t}$ are equi-Lipshitz because the functions $y_{t}$, $t \geqslant t_{0}^{\prime}$ are equi-Lipshitz. As a consequence, both entropy solutions $\omega$ and $y$ are continuous on $\left[t_{0}^{\prime}, \infty\right) \times \mathbb{T}$; hence, $\left\|y_{t}-\omega_{t}\right\|_{L^{1}}$ is constant and thus is zero. We have proved that $y=\omega$ on $\left[t_{1}, \infty\right) \times \mathbb{T}$.

\subsection{0 .}

The fact that backward characteristics always exist, but that forward characteristics do not, is one of the key features of entropy solutions. Particles may be absorbed by shocks, but they cannot be created by shocks. In order to understand the full future of a given particle, it is useful to introduce the notion of a weak characteristic. Consider an entropy solution $y(t, x):\left[t_{0}, \infty\right) \times \mathbb{T} \longrightarrow \mathbb{R}$ of the Burgers equation, let $c=\int_{\mathbb{T}} y \mathrm{~d} x$ and let $u(t, x):\left[t_{0}, \infty\right) \times \mathbb{T} \longrightarrow \mathbb{R}$ be a viscosity solution of $(H J c)$ such that $y=c+\partial_{x} u$. We say 
that an absolutely continuous curve $x(s):\left[t_{0}, \infty\right) \supset\left[t, t^{\prime}\right] \longrightarrow \mathbb{T}$ is a weak $y$-characteristic if it satisfies the equation

$$
\dot{x}(s) \in\left[\partial_{p} H\left(s, x(s), y_{s}^{-}(x(s))\right), \partial_{p} H\left(s, x(s), y_{s}^{+}(x(s))\right)\right]
$$

almost everywhere. It is clear that $y$-characteristics are weak $y$-characteristics, and that the notions of weak $y$-characteristics and of $y$-characteristics coincide if $y$ is a continuous solution.

Proposition. For each $t>t_{0}$ and $x_{0} \in \mathbb{T}$, there exists one and only one weak characteristic $x(s):[t, \infty) \longrightarrow \mathbb{T}$ satisfying $x(t)=x_{0}$.

Proof. In view of the convexity of $H$ in the $p$ variable, there exists a constant $K$ such that the function $Y(s, x)=\partial_{p} H(s, x, y(s, x))$ satisfies $|Y(s, x)| \leqslant K$ and the Oleinik inequality

$$
Y(s, x+\delta) \leqslant Y(s, x)+K \delta
$$

for $\delta>0, s \geqslant t$. Since $\partial_{p} H\left(s, x, y^{ \pm}(s, x)\right)=Y^{ \pm}(s, x)$, the equation for weak characteristics can be written as

$$
\dot{x}(s) \in\left[Y^{-}(x(s)), Y^{+}(x(s))\right] .
$$

Now the proposition follows from a result of Filippov, see $[\mathrm{H}]$, theorem 1.4.2.

3.11 .

Proposition. All weak y-characteristics $x(s):[t, \infty) \longrightarrow \mathbb{T}$ have the same rotation number

$$
\lim \frac{1}{t} \int_{t_{0}}^{t} \dot{x}=\rho(c)=\alpha^{\prime}(c) .
$$

Proof. Let $\left.x(t):] t_{0}, \infty\right) \longrightarrow \mathbb{T}$ and $\left.\left.q(t):\right] t_{0}, \infty\right) \longrightarrow \mathbb{T}$ be, respectively, a genuine $y$-characteristic and a weak $y$-characteristic. Let $\left.\bar{x}(t):] t_{0}, \infty\right) \longrightarrow \mathbb{R}$ and $\left.\left.\bar{q}(t):\right] t_{0}, \infty\right) \longrightarrow \mathbb{R}$ be continuous functions such that $\bar{x}(t) \bmod 1=x(t)$ and $\bar{q}(t) \bmod 1=q(t)$, and such that $\bar{x}\left(t_{0}\right) \leqslant \bar{q}\left(t_{0}\right) \leqslant \bar{x}\left(t_{0}\right)+1$. Note that $\bar{q}(t)=\bar{q}\left(t_{0}\right)+\int_{t_{0}}^{t} \dot{q}$, with a similar property for $\bar{x}$. The uniqueness of weak $y$-characteristics implies that $\bar{x}(t) \leqslant \bar{q}(t) \leqslant \bar{x}(t)+1$ for all $t$. Since $x$ is a genuine $y$-characteristic, it is calibrated by $u$, so that proposition 2.7 implies

$$
\frac{\bar{x}(t)}{t} \longrightarrow \rho(c) \quad \text { and hence } \quad \frac{\bar{q}(t)}{t} \longrightarrow \rho(c) .
$$

\section{Pairs of solutions and dissipation}

It is well known that if $y$ and $z$ are entropy solutions of $(B)$, then the norm $\left\|y_{t}-z_{t}\right\|_{L^{1}}$ is a non-increasing function of $t$. We shall give a proof of this fact. In short, it is a consequence of the fact that values of local maxima of differences of viscosity solutions are decreasing. We now give the details.

\section{1.}

Let $f: \mathbb{T} \longrightarrow \mathbb{R}$ be a continuous function. We say that $x_{0} \in \mathbb{T}$ is a point of local maximum of $f$ if there exists an interval $] a, b\left[\subset \mathbb{T}\right.$ containing $x_{0}$ and such that $f\left(x_{0}\right)=\max _{] a, b[} f$ and 
$f\left(x_{0}\right)>\max (f(a), f(b))$. The value $f\left(x_{0}\right)$ is then called a value of local maximum. The following proposition states that the values of local maxima of a difference $u_{t}-v_{t}$ of viscosity solutions are non-increasing functions of $t$.

Proposition. Let $u, v:\left[t_{0}, \infty\right) \times \mathbb{T} \longrightarrow \mathbb{R}$ be a pair of viscosity solutions of $(H J c)$, let $t_{1}>t_{0}$, and let $d=u-v$. Let $x_{1}$ be a point of local maximum for $d_{t_{1}}$ and let $] a, b[$ be an open interval containing $x_{1}$, and such that $\max \left(d_{t_{1}}(a), d_{t_{1}}(b)\right)<d\left(t_{1}, x_{1}\right)=\max _{] a, b[} d_{t_{1}}$. Let $a(t)$ and $b(t)$ be curves calibrated by $u$ and satisfying $a\left(t_{1}\right)=a, b\left(t_{1}\right)=b$. Then for each $t$ in $\left.] t_{0}, t_{1}\right]$, we have $M(t)=\max _{[a(t), b(t)]} d_{t}>\max (d(t, a(t)), d(t, b(t)))$; hence, $M(t)$ is a value of local maximum. In addition, the value $M(t)$ is a continuous and non-increasing function of $t$ on ]$\left.t_{0}, t_{1}\right]$.

Proof. The functions $d(t, a(t))$ and $d(t, b(t))$ are non-decreasing on $\left[t_{0}, t_{1}\right]$; hence, $d(t, a(t)) \leqslant d\left(t_{1}, a\left(t_{1}\right)\right)<M\left(t_{1}\right)$ and $d(t, b(t)) \leqslant d\left(t_{1}, b\left(t_{1}\right)\right)<M\left(t_{1}\right)$. On the other hand, consider a curve $q(t)$ that is calibrated by $v$ and satisfies the final condition $q\left(t_{1}\right)=x_{1}$. Since the function $d(t, q(t))$ is non-increasing, we have $d(t, q(t)) \geqslant d\left(t_{1}, q\left(t_{1}\right)\right)=M\left(t_{1}\right)$. It follows that $d(t, q(t))>d(t, a(t))$ and $d(t, q(t))>d(t, b(t))$ for each $t$; hence, the curve $q(t)$ cannot cross the curves $a(t)$ and $b(t)$, and $q(t) \in] a(t), b(t)$ [ for each $t$. As a consequence, we have $M(t) \geqslant d(t, q(t))>\max (d(t, a(t)), d(t, b(t)))$ and thus $M(t)$ is a value of local maximum. In addition, we have $M(t) \geqslant d(t, q(t)) \geqslant M\left(t_{1}\right)$. The same construction can be performed with a smaller final time $t_{1}^{\prime}$ satisfying $t<t_{1}^{\prime} \leqslant t_{1}$; hence, we have the inequality $M(t) \geqslant M\left(t_{1}^{\prime}\right)$ for $t<t_{1}^{\prime} \leqslant t_{1}$. As a consequence, the function $M(t)$ is non-increasing on ]$\left.t_{0}, t_{1}\right]$.

\section{2.}

In order to apply the proposition to pairs of entropy solution of $(B)$ with possibly different averages, we need some refinements. From now on, we sometimes consider functions on $\mathbb{T}$ as periodic functions on $\mathbb{R}$ without changing their names.

Corollary. Let $c$ and $c^{\prime}$ be two real numbers, let $\left.\left.u:\right] t_{0}, \infty\right) \times \mathbb{T} \longrightarrow \mathbb{R}$ be a viscosity solution of $(H J c)$, let $\left.v:] t_{0}, \infty\right) \times \mathbb{T} \longrightarrow \mathbb{R}$ be a viscosity solution of $\left(H J c^{\prime}\right)$, and let $t_{1}>t_{0}$. We denote by $d_{t}$ the difference $d_{t}(x)=u_{t}(x)-v_{t}(x)+\left(c-c^{\prime}\right) x: \mathbb{R} \longrightarrow \mathbb{R}$. Let $x_{1} \in \mathbb{R}$ be a point of local maximum for $d_{t_{1}}$, and let $] a, b[\subset \mathbb{R}$ be an open interval of length less than 1 containing $x_{1}$, and such that $\max \left(d_{t_{1}}(a), d_{t_{1}}(b)\right)<d\left(t_{1}, x_{1}\right)=\max _{] a, b[} d_{t_{1}}$. Let $a(t)$ and $b(t)$ be curves calibrated by $u($ for $(H J c))$ and satisfying $a\left(t_{1}\right)=a, b\left(t_{1}\right)=b$. Then there exists $t_{0}^{\prime}<t_{1}$ such that, for $t \in\left[t_{0}^{\prime}, t_{1}\right]$, we have $M(t)=\max _{] a(t), b(t)[} d_{t}>\max (d(t, a(t)), d(t, b(t)))$. The value $M(t)$ is a continuous and non-increasing function of $t$ on $\left[t_{0}^{\prime}, t_{1}\right]$. The functions $t \longmapsto d(t, a(t))$ and $t \longmapsto d(t, b(t))$ are non-decreasing on $\left[t_{0}^{\prime}, t_{1}\right]$.

Addendum. If $] a^{\prime}, b^{\prime}[\subset] a, b\left[\right.$ is a smaller interval and if $M^{\prime}=d\left(t_{1}, x_{1}^{\prime}\right)=\max _{] a^{\prime}, b^{\prime}[} d_{t_{1}}>$ $\max \left(d_{t_{1}}\left(a^{\prime}\right), d_{t_{1}}\left(b^{\prime}\right)\right)$, then the corollary can be applied to the value $M^{\prime}$ in the interval $] a^{\prime}, b^{\prime}[$ with the same time $t_{0}^{\prime}$.

Proof. Let us fix intervals $I$ and $J$ of length less than 1 such that $[a, b] \subset I \subset \bar{I} \subset J$. We also call $I$ and $J$ the images of $I$ and $J$ in $\mathbb{T}$, which are proper subsets of $\mathbb{T}$. Let $K^{\prime}$ be a common Lipschitz constant of the functions $v_{t}, t \geqslant\left(t_{0}+t_{1}\right) / 2$, and let $K=2 K^{\prime} /(1-|J|)$. Let us fix a time $t_{0}^{\prime}<t_{1}$ and a 1-periodic $K$-Lipschitz function $w_{t_{0}^{\prime}}: \mathbb{R} \longrightarrow \mathbb{R}$ such that $w_{t_{0}^{\prime}}(x)=v\left(t_{0}^{\prime}, x\right)+\left(c^{\prime}-c\right) x$ for $x \in \mathbb{Z}+J$. We can see $w_{t_{0}^{\prime}}$ as a function on $\mathbb{T}$ and define in 
a natural way $w(t, x)=V_{t_{0}^{\prime}, t}^{c}\left(w_{t_{0}^{\prime}}\right)(x)$ for $t \geqslant t_{0}^{\prime}$. We claim that $w(t, x)=v(t, x)+\left(c^{\prime}-c\right) x$ for all $x \in I$ and $t \in\left[t_{0}^{\prime}, t_{1}\right]$, if $t_{0}^{\prime}<t_{1}$ is large enough. Assuming the claim, and assuming in addition that $t_{0}^{\prime}$ is sufficiently large that the curves $a(t)$ and $b(t)$ remain in $I$ on $\left[t_{0}^{\prime}, t_{1}\right]$, the corollary follows from the proposition above applied to $u$ and $w$, which both solve $(H J c)$. In order to prove the claim, recall that

$$
w(t, x)=\min \left(w_{t_{0}^{\prime}}\left(x\left(t_{0}^{\prime}\right)\right)+\int_{t_{0}^{\prime}}^{t} L(s, x(s), \dot{x}(s))-c \dot{x}(s) \mathrm{d} s\right)
$$

and

$$
v(t, x)=\min \left(v_{t_{0}^{\prime}}\left(x\left(t_{0}^{\prime}\right)\right)+\int_{t_{0}^{\prime}}^{t} L(s, x(s), \dot{x}(s))-c^{\prime} \dot{x}(s) \mathrm{d} s\right),
$$

where the minima are taken on the set of $C^{1}$ curves $x(s):\left[t_{0}^{\prime}, t\right] \longrightarrow \mathbb{R}$ terminating at $x$. By superlinearity of $L$, it is possible to choose $t_{0}^{\prime}$ so close to $t_{1}$ that, if $t_{0}^{\prime} \leqslant t \leqslant t^{\prime} \leqslant t_{1}$ and $x(s):\left[t, t^{\prime}\right] \longrightarrow \mathbb{R}$ is a curve starting outside $J$ and ending inside $I$, then

$\int_{t}^{t^{\prime}} L(s, x(s), \dot{x}(s)) \mathrm{d} s \geqslant 2 K+\int_{t}^{t^{\prime}} L\left(s, x\left(t^{\prime}\right), 0\right) \mathrm{d} s+\left(|c|+\left|c^{\prime}\right|\right) \int_{t}^{t^{\prime}}|\dot{x}(s)| \mathrm{d} s$.

If $t_{0}^{\prime}$ is chosen sufficiently close to $t_{1}$, then for each $x \in I$, the curves reaching the minima in the expressions of $w$ and $v$ above satisfy $x(s) \in J$ for all $s$. We have proved that if $t_{0}^{\prime}$ is large enough, then for each $x \in I$ it is possible to restrict the minima in the expression for $w$ and $v$ above to curves $x(s)$ contained in $J$. For such a curve, we have

$$
\begin{aligned}
& w_{t_{0}^{\prime}}\left(x\left(t_{0}^{\prime}\right)\right)+\int_{t_{0}^{\prime}}^{t} L(s, x(s), \dot{x}(s))-c \dot{x}(s) \mathrm{d} s \\
& \quad=v_{t_{0}^{\prime}}\left(x\left(t_{0}^{\prime}\right)\right)+\left(c^{\prime}-c\right) x\left(t_{0}^{\prime}\right)+c\left(x\left(t_{0}^{\prime}\right)-x\right)+\int_{t_{0}^{\prime}}^{t} L(s, x(s), \dot{x}(s)) \mathrm{d} s \\
& \quad=v_{t_{0}^{\prime}}\left(x\left(t_{0}^{\prime}\right)\right)+\left(c^{\prime}-c\right) x+\int_{t_{0}^{\prime}}^{t} L(s, x(s), \dot{x}(s))-c^{\prime} \dot{x}(s) \mathrm{d} s .
\end{aligned}
$$

As a consequence, the equality $w(t, x)=\left(c^{\prime}-c\right) x+v(t, x)$ holds for all $t \in\left[t_{0}^{\prime}, t_{1}\right]$ and all $x \in J$. This ends the proof of the claim and the proof of the corollary.

4.3 .

Theorem. Let $y(t, x)$ and $\left.z(t, x):] t_{0}, \infty\right) \times \mathbb{T} \longrightarrow \mathbb{R}$ be two entropy solutions of $(B)$. Then the function

$$
t \longmapsto\left\|y_{t}-z_{t}\right\|_{1}
$$

is non-increasing. In addition, let $x_{0}(t)$ and $x_{1}(t)$ be two curves that are characteristics both for $y$ and $z$, and let $J(t)$ and $I(t)$ be the two continuously varying intervals of $\mathbb{T}$ such that $I(t) \cup J(t)=\mathbb{T}$ and $I(t) \cap J(t)=\left\{x_{0}(t), x_{1}(t)\right\}$. Then each of the functions

$$
t \longmapsto\left\|\left(y_{t}-z_{t}\right)_{\mid I(t)}\right\|_{1} \quad \text { and } \quad t \longmapsto\left\|\left(y_{t}-z_{t}\right)_{\mid J(t)}\right\|_{1}
$$

is non-increasing.

Proof. We shall prove the first part, leaving the easy modifications needed to prove the second part to the reader. Let $y=c+\partial_{x} u$ and $z=c^{\prime}+\partial_{x} v$ be two entropy solutions of $(B)$, and let $d(t, x)=u_{t}(x)-v_{t}(x)+\left(c-c^{\prime}\right) x$. The idea of the proof is very simple. The $L^{1}$ norm of 
$y_{t}-z_{t}$ is the total variation of the function $d_{t}$. Since the local maxima of this function do not increase, and since its local minima do not decrease, the total variation of $d_{t}$ cannot increase. The details are, however, a bit tedious.

We consider $u$ and $v$ as periodic functions $\left.] t_{0}, \infty\right) \times \mathbb{R} \longrightarrow \mathbb{R}$ and define $d(t, x)=$ $u_{t}(x)-v_{t}(x)+\left(c-c^{\prime}\right) x$. We denote by $\operatorname{Var}\left(d_{t}\right)$ the total variation of $d_{t}$ on any interval of length 1 . This quantity does not depend on the interval, and

$$
\operatorname{Var}\left(d_{t}\right)=\left\|y_{t}-z_{t}\right\|_{L^{1}(\mathbb{T})} .
$$

We shall prove that, for each $t_{1}>t_{0}$, there exists a time $\left.t_{0}^{\prime} \in\right] t_{0}, t_{1}[$ such that, for each $t \in] t_{0}^{\prime}, t_{1}[$, we have $\operatorname{Var}\left(d_{t}\right) \geqslant \operatorname{Var}\left(d_{t_{1}}\right)$. Because it is also continuous, the function $t \longmapsto \operatorname{Var}\left(d_{t}\right)$ is then non-increasing, which is the desired result.

Let us fix a time $t_{1}>t_{0}$. Assume first that the function $d_{t_{1}}$ does not have any local minimum. In this case, $\operatorname{Var}\left(d_{t_{1}}\right)=\left|c^{\prime}-c\right|$. On the other hand, the inequality $\operatorname{Var}\left(d_{t}\right) \geqslant\left|c^{\prime}-c\right|$ clearly holds for all $t$; hence, we have proved that

$$
\operatorname{Var}\left(d_{t}\right) \geqslant \operatorname{Var}\left(d_{t_{1}}\right)
$$

for all $t<t_{1}$.

Otherwise, there exists a point $a \in \mathbb{R}$ of local minimum of $d_{t_{1}}$. We consider a $y$-characteristic $\left.a(t):] t_{0}, t_{1}\right] \longrightarrow \mathbb{R}$ terminating at $a$. There exist non-decreasing sequences $\mathcal{M}_{k}^{-}$and $\mathcal{M}_{k}^{+}$of finite subsets of $] a, a+1[$ such that for each $k$ :

- Each point of $\mathcal{M}_{k}^{-}$is a point of local minimum of $d_{t_{1}}$.

- Each maximal interval in the complement $] a, a+1\left[-\mathcal{M}_{k}^{-}\right.$of $\mathcal{M}_{k}^{-}$contains exactly one point of $\mathcal{M}_{k}^{+}$.

- Each point of $\mathcal{M}_{k}^{+}$is a point of local maximum of $d_{t_{1}}$.

- The sets $\mathcal{M}_{k}^{-}$and $\mathcal{M}_{k}^{+}$are disjoint.

- We have, when $k \longrightarrow \infty$,

$$
2 \sum_{x \in \mathcal{M}_{k}^{+}} d_{t_{1}}(x)-2 \sum_{x \in \mathcal{M}_{k}^{-}} d_{t_{1}}(x)-d_{t_{1}}\left(a\left(t_{1}\right)\right)-d_{t_{1}}\left(a\left(t_{1}\right)+1\right) \longrightarrow \operatorname{Var}\left(d_{t_{1}}\right) .
$$

It is possible that $\mathcal{M}_{k}^{-}$is empty for all $k$. In this case, $\mathcal{M}_{+}^{k}$ is also independent of $k$ and contains a single point $x$, and we have

$$
\operatorname{Var}\left(d_{t_{1}}\right)=2 d_{t_{1}}(x)-d_{t_{1}}\left(a\left(t_{1}\right)\right)-d_{t_{1}}\left(a\left(t_{1}\right)+1\right) .
$$

In view of the corollary, there exists $t_{0}^{\prime}$ such that the function $M(t)=\max _{] a(t), a(t+1)[} d_{t}$ is non-increasing on $\left[t_{0}^{\prime}, t_{1}\right]$, and the functions $d(t, a(t))$ and $d(t, b(t))$ are non-decreasing on this interval. As a consequence, for $t \in] t_{0}, t_{1}[$, we have

$\operatorname{Var}\left(d_{t}\right) \geqslant 2 M(t)-d_{t}(a(t))-d_{t}(a(t)+1) \geqslant 2 M\left(t_{1}\right)-d_{t_{1}}\left(a\left(t_{1}\right)\right)-d_{t_{1}}\left(a\left(t_{1}\right)+1\right)=\operatorname{Var}\left(d_{t_{1}}\right)$.

Otherwise, the function $d_{t_{1}}$ has oscillations and the sets $\mathcal{M}_{k}^{-}$are not empty. Let us choose, for each point $x \in \mathcal{M}_{k}^{-}$, a $y$-characteristic $x(t):\left[t_{0}, t_{1}\right] \longrightarrow \mathbb{R}$ satisfying $x\left(t_{1}\right)=x$. Let $\mathcal{M}_{k}^{-}(t)$ be the union of all the points $x(t)$ obtained that way. The corollary above and its addendum allow to find a time $\left.t_{0}^{\prime} \in\right] t_{0}, t_{1}$ [ independent of $k$ such that, if ] $x, x^{\prime}[\subset] a, a+1[$ is a maximal interval of the complement of $\mathcal{M}_{k}^{-}$, then the $y$-characteristics $x(t)$ and $x^{\prime}(t)$ associated with $x$ and $x^{\prime}$ satisfy $a(t) \leqslant x(t)<x^{\prime}(t) \leqslant a(t)+1$ for each $\left.t \in\right] t_{0}^{\prime}, t_{1}[$, and the interval ] $x(t), x^{\prime}(t)$ [ contains a point of local maximum $q(t)$ of $d_{t}$, such that the associated value of local maximum $d(t, q(t))$ is non-increasing. Let $\mathcal{M}_{k}^{+}(t)$ be the union of all such points $q(t)$. 
Recall also, from the corollary, that the function $t \longmapsto d(t, x(t))$ is non-decreasing on $] t_{0}^{\prime}, t_{1}[$ for each of the chosen characteristics $x(t) \in \mathcal{M}_{k}^{-}(t)$. We have, for $t \in\left[t_{0}^{\prime}, t_{1}\right]$

$$
\begin{aligned}
\operatorname{Var}\left(d_{t}\right) & \geqslant 2 \sum_{q \in \mathcal{M}_{k}^{+}(t)} d_{t}(q)-2 \sum_{x \in \mathcal{M}_{k}^{-}(t)} d_{t}(x)-d_{t}(a(t))-d_{t}(a(t)+1) \\
& \geqslant 2 \sum_{q \in \mathcal{M}_{k}^{+}\left(t_{1}\right)} d_{t_{1}}(q)-2 \sum_{x \in \mathcal{M}_{k}^{-}\left(t_{1}\right)} d_{t_{1}}(x)-d_{t_{1}}\left(a\left(t_{1}\right)\right)-d_{t_{1}}\left(a\left(t_{1}\right)+1\right) \longrightarrow \operatorname{Var}\left(d_{t_{1}}\right) ;
\end{aligned}
$$

hence, $\operatorname{Var}\left(d_{t}\right) \geqslant \operatorname{Var}\left(d_{t_{1}}\right)$.

\section{Acknowledgments}

Most of this work was written while I was staying for one year in EPFL, Switzerland, with a grant of the Swiss National Fund for research. This period was very pleasant and fruitful. I would like to thank Boris Buffoni for proposing me for this opportunity, and for his interest in the present work.

\section{References}

[A] Arnold V I 1980 Chapitres supplémentaires de la théorie des équations différentielles ordinaires Grundlehren der mathematischen Wissenschaften 2nd edn, vol 250 (Berlin: Springer)

Arnold V I 1988 Geometrical Methods in the Theory of Ordinary Differential Equations (Engl. transl. from the Russian)

[Ba] Barles G 1994 Solutions de viscosité des equations de Hamilton-Jacobi Mathematiques \& Application vol 17 (Berlin: Springer)

[Be] Bernard P 2002 Connecting orbits of time dependent Lagrangian systems Ann. Inst. Fourier 52 1533-68

[BR] Bernard P and Roquejoffre J M 2004 Convergence to time-periodic solutions in Hamilton-Jacobi equations on the circle Commun. PDE 29 457-69

[B] Bessi U 2003 Aubry-Mather theory and Hamilton-Jacobi equations Commun. Math. Phys. 235 495-511

[Br] Brezis H 1983 Analyse Fonctionnelle (Paris: Masson)

[E] E W 1999 Aubry-Mather theory and periodic solutions of the forced Burgers equation Commun. Pure Appl. Math. 52 811-28

[EG] Evans L C and Gariepy R E 1992 Measure Theory and Fine Properties of Functions (Studies in Advanced Mathematics) (Boca Raton, FL: CRC Press)

[Fa1] Fathi A 1997 Théorème KAM faible et théorie de Mather sur les systèmes lagrangiens [A weak KAM theorem and Mather's theory of Lagrangian systems] C. R. Acad. Sci. I Math. 324 1043-6 (in French)

[Fa2] Fathi A in preparation

[Fa3] Fathi A 1998 Sur la convergence du semi-groupe de Lax-Oleinik [Convergence of the Lax-Oleinik semigroup] C. R. Acad. Sci. I Math 327 267-70 (in French)

[FM] Fathi A and Mather J N 2000 Failure of convergence of the Lax-Oleinik semi-group in the time-periodic case Bull. Soc. Math. France 128 473-83

[FMa] Fathi A and Maderna E 2002 Weak KAM theorem on non compact manifolds Preprint

[Fl] Fleming W H 1969 The Cauchy problem for a nonlinear first order partial differential equation J. Diff. Eqns 5 515-30

[G] Gomes D 2002 Viscosity solutions of Hamilton-Jacobi equations, and asymptotics for Hamiltonian systems Calc. Var. PDE 14.3 345-57

[HF] Herman M R 1983 Sur les courbes invariantes par les difféomorphismes de l'anneau vol 1 (On the curves invariant under diffeomorphisms of the annulus. vol 1) With an appendix by Albert Fathi Astérisque 103-104 (in French)

[H] Hörmander L 1997 Lectures on Nonlinear Hyperbolic Differential Equations (Mathematique Application vol 26) (Berlin: Springer)

[JKM] Jauslin H R, Kreiss O and Moser J 1999 On the forced Burgers equation with periodic boundary conditions Proc. Symp. Pure. Math. 65 133-53

[KO] Katznelson Y and Ornstein D S 1997 Twist maps and Aubry-Mather sets Contemp. Math. 211 343-57

[L] Lax P 1957 Hyperbolic systems of conservation laws II Commun. Pure Appl. Maths 10 537-66 
[Ma1] Mather J N 1991 Variational construction of orbits of twist diffeomorphisms J. Am. Math. Soc. 4 207-63

[Mo] Moser J 1986 Monotone twist mappings and the calculus of variations Ergod. Theory Dyn. Syst. 6 401-13

[Ro] Roquejoffre J-M 2001 Convergence to steady states or periodic solutions in a class of Hamilton-Jacobi equations J. Math. Pure Appl. 80 85-104

[So] Sobolevskii A N 1999 Periodic solutions of the Hamilton-Jacobi equation with a periodic nonhomogeneity, and the Aubry-Mather theory Mat. Sb. 190 87-104 (Russian)

Sobolevskii A N 1999 Sb. Math. 190 1487-504 (Translation)

[Se] Serre D 1996 Systèmes de lois de conservation vol 1 (Paris: Diderot)

[Si] Siburg K F 1998 A dynamical approach to Birkhoff's theorem Ens. Math. 44 291-303 